\title{
NONDESTRUCTIVE TESTING USED ON TIMBER IN SPAIN: A LITERATURE REVIEW
}

\author{
Daniel F. Llana ${ }^{1,2, \$}$, Guillermo Íñiguez-González ${ }^{1,3}$, M. Rafael Díez ${ }^{1,4}$, Francisco Arriagal ${ }^{1,3}$
}

\begin{abstract}
Nondestructive testing (NDT) includes several highly efficient techniques for the estimation of the physical and mechanical properties of structural timber. Apart from visual grading, scientific research using Nondestructive testing on timber has been used in Spain since the 1990s. Nondestructive testing can be used for two different purposes: timber grading and the assessment of existing timber structures. The most common devices used in Spain are portable ones based on ultrasound, stress waves, vibration and probing techniques. Many statistical linear models for estimating the mechanical properties of new sawn timber and timber from existing structures have been proposed. Furthermore, several factors that affect Nondestructive testing measurements have been studied (moisture content, temperature, specimen dimensions, sensors position-grain angle, among others) and adjustment factors have also been proposed. Species have been characterized for visual grading standards from the 1980s to date. The large number of research works using different species, devices and procedures shows the need of homogenization and standardization of Nondestructive testing use. This paper presents a review of research works using Nondestructive testing on timber in Spain, in order to add to knowledge, elucidate the concepts to unify Nondestructive testing used and promote research group collaboration in the near future.
\end{abstract}

Keywords: Acoustic techniques, nondestrucyive testing, stress waves, structural timber, ultrasound waves, vibration techniques.

\section{INTRODUCTION}

Scientific research into the determination of timber mechanical properties began in Spain in the 1960s, in the INIA Structural Timber Laboratory (Figure 1a). Arriaga et al. (1992) published the first scientific research work using Nondestructive Testing (NDT) on timber in Spain. The Steinkamp BP-V (BPV), a portable ultrasound device with exponential tip $50 \mathrm{kHz}$ sensors, was used on 34 pieces from existing structures to estimate their mechanical properties with determination coefficients $\left(\mathrm{R}^{2}\right)$ between the modulus of elasticity (MOE) and the dynamic modulus of elasticity (Edyn) of $37 \%$ (Figure 1b). Martínez (1992) used the same NDT device on structural maritime pine timber (40 $\mathrm{mm}$ x $100 \mathrm{~mm}$ and $50 \mathrm{~mm}$ x $150 \mathrm{~mm}$ ) in his $\mathrm{PhD}$ thesis. Bucur et al. (1993) presented the first SCI JCR publication in Spain of NDT on timber using the BPV and X-ray for fungal decay detection in pine and European beech. Several other works were presented with a focus on detecting decay and defects using ultrasound waves (Palaia et al. 1993, Galvañ et al. 1994, Martín 1994, Troya and Navarrete 1994). Rodríguez-Liñán and Rubio (1995) and Rubio (1997) estimated MOE and bending strength (MOR)

\footnotetext{
${ }^{1}$ Timber Construction Research Group, Universidad Politécnica de Madrid, Madrid, Spain.

${ }^{2}$ Timber Engineering Research Group, National University of Ireland Galway, Galway, Ireland.

${ }^{3}$ Department of Forestry and Environmental Engineering and Management, Montes (School of Forest Engineering and Natural Resources), Universidad Politécnica de Madrid, Madrid, Spain.

${ }^{4}$ Structural Timber Laboratory, Forest Products Department, INIA-CIFOR, Madrid, Spain.

•Corresponding author: danielfllana@gmail.com

Received: 01.01.2019 Accepted: 01.12.2019
} 
of new Scots pine timber, timber from existing structures and small clear specimens using the BPV with a $\mathrm{R}^{2}$ from $36 \%$ to $44 \%$ for MOE and MOR, respectively. Pedras et al. (1997) estimated MOE from velocity, with a $\mathrm{R}^{2}$ of $81 \%$ in small clear sweet chestnut specimens. Palaia et al. (2000) proposed models for density $(\rho)$ estimation from needle penetration resistance (NPR) depth using the Pilodyn with a $\mathrm{R}^{2}$ of $80 \%$ using small clear specimens of Scots, maritime and Caribbean pitch pine. Riesco (2001) used BPV velocity to estimate the MOE of small clear specimens of European oak with a $40 \% \mathrm{R}^{2}$. At the end of the 1990s an automatic bending classification machine, the Cook Bolinders (SG-AF Tecmach Ltd., St. Albans, UK), arrived in the INIA Structural Timber Laboratory (Figure 1c). Hermoso (2001) reported the settings used to classify Spanish Scots pine with this machine, and Conde (2003) presented the settings for Salzmann pine. Furthermore, both doctoral theses also estimated structural timber MOE and MOR from ultrasound wave velocity using the Sylvatest (Syl) portable device combined with visual grading parameters.

\section{Acoustic techniques (ultrasound and stress waves)}

Esteban (2003) used BPV and Sylvatest Duo (SylDuo) measurements combined with visual parameters to estimate the mechanical properties of Scots and maritime pine from existing structures. Hermoso et al. (2003) compared grading results using the Syl and Cook Bolinders, obtaining a lower rejection percentage with the latter for Scots and Salzmann pine. Arriaga et al. (2006) reported a $\mathrm{R}^{2}$ of $73 \%$ when estimating MOE from SylDuo velocity in missanda. Capuz et al. (2007) estimated a C18 strength class based on in-situ SylDuo measurements in the timber structured historic building "Lonja de Mercaderes" in Valencia. Hermoso et al. (2007) studied Salzmann pine round small-diameter timber, estimating MOE from Edyn with a $68 \% \mathrm{R}^{2}$. Íñiguez-González (2007) used ultrasound on large cross-section radiata, Scots and Salzmann pine timber (150 $\mathrm{mm} \times 200 \mathrm{~mm}, 200 \mathrm{~mm}$ x $250 \mathrm{~mm}$ ) to estimate their properties. Palaia et al. (2008) presented a procedure for the assessment of timber structures using several NDT techniques, testing them on Scots pine from existing structures. Basterra et al. (2009) evaluated historic buildings in "Chinchón Plaza Mayor" using ultrasound and probing techniques. Carballo et al. (2009a), Carballo et al. (2009b) presented a review of 30 years of NDT, together with an estimation of maritime pine MOE using the SylDuo and MicroSecond Timer (MST) velocity with a $\mathrm{R}^{2}$ of $55 \%$ and $70 \%$ with the Edyn. In the case of MOR, a $\mathrm{R}^{2}$ of $39 \%$ was found when a knottiness parameter was included. Esteban et al. (2009) estimated MOE and MOR by stress waves and probing methods using the Íñiguez-González (2007) models, and assigned a strength class in the assessment of the Valsaín sawmill historic building (Figure 1d). Atienza-Conejo (2012) used pulse-echo ultrasound to detect xylophage insect attack in timber ships. Casado et al. (2012) estimated the MOE of black poplar timber by combining SylDuo velocity and visual parameters with a $\mathrm{R}^{2}$ of $68 \%$. Montón (2012) tested Catalonian radiata pine, estimating its properties with ultrasound and stress waves. Vega et al. (2012) estimated the mechanical properties of sweet chestnut using the Sylvatest Trio (SylTrio) and MST, obtaining a $\mathrm{R}^{2}$ of $70 \%$ using Edyn or velocity and density. However, MOR was estimated with a $\mathrm{R}^{2}$ of $27 \%$ even when a knottiness parameter was included. Merlo et al. (2014) used the IML Micro Hammer (IML MH) device (IML, Wiesloch, Germany) on standing maritime pine trees estimating the MOE of sawn boards from these trees with a $\mathrm{R}^{2}$ of $55 \%$. Vázquez et al. (2015) used 13 polyhedral small clear specimens of sweet chestnut to determine Young's moduli, shear moduli and Poisson's ratios by ultrasound with $1 \mathrm{MHz}$ sensors, finding a good correlation with MOE of structural timber. Vilches et al. (2015) assigned strength classes C14 and C18 to Scots pine beams from an existing structure by stress waves using the Íñiguez-González (2007) models. Abián and Segura (2016) estimated the residual capacity of fire-damaged Scots pine timber from existing structures using the ultrasound wave method. Llana (2016) used the USLab device with $45 \mathrm{kHz}$ sensors to estimate MOE from Edyn with a $\mathrm{R}^{2}$ of $90 \%$. Crespo et al. (2017) tested small clear specimens of southern blue gum with $1 \mathrm{MHz}$ ultrasound sensors to obtain their elastic values. Morales-Conde and Machado (2017) used PUNDITplus (Proceq, Schwerzenbach, Switzerland) with $54 \mathrm{kHz}$ sensors and MST on 30 clear wood pieces of maritime pine to estimate MOE from Edyn. Higher $\mathrm{R}^{2}$ (91 \%) combining MST measurements at different depths than using PUNDIT (71\%) was found. Hillig et al. (2018) used SylDuo, USLab and MST devices to study wood-polymer-composites in the Universidad Politécnica de Madrid Timber Laboratory. Osuna-Sequera et al. (2019a) studied several criteria to determine the cross-section in existing timber structures to estimate MOE from Edyn. Vega et al. (2019a) estimated MOE of 216 dry sweet chestnut small-diameter logs using MST velocity and Edyn with $\mathrm{R}^{2}$ of $64 \%$ and $67 \%$, respectively and a grading system was designed based on MST velocity. 


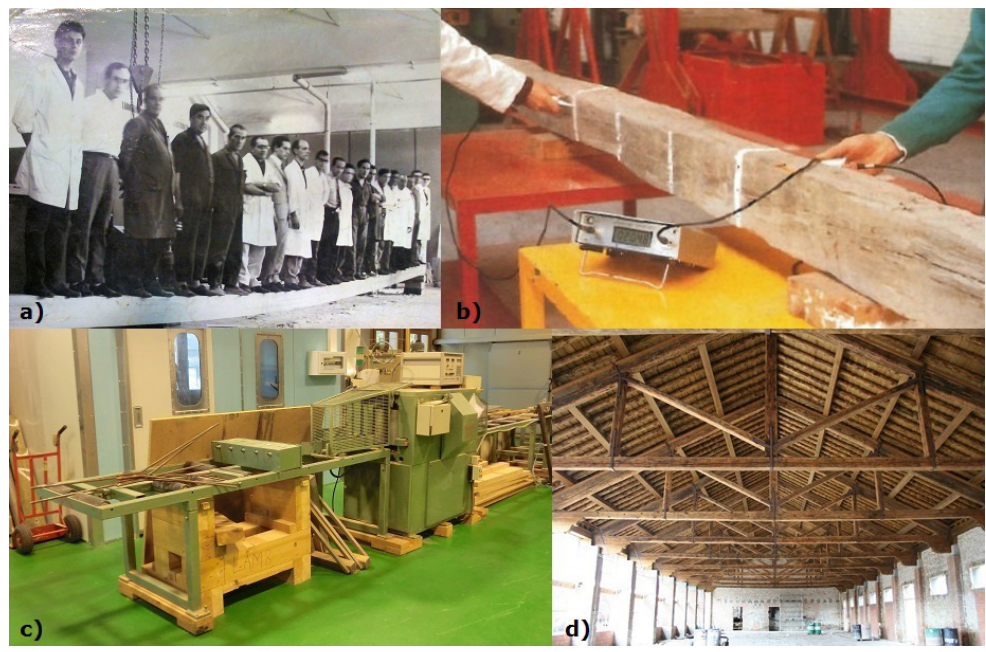

Figure 1: Spanish scientific timber research facts: a) INIA Structural Timber Laboratory in the1960s and 1970s. b) Arriaga et al. (1992) ultrasound measurements. c) Cook Bolinders, INIA Structural Timber Laboratory. d) Valsaín sawmill historic building.

\section{Vibration techniques}

Arriaga et al. (2005a) published the first scientific research work done in Spain with vibration technique to grade 75 radiata pine specimens using the Portable Lumber Grader (PLG). Broto et al. (2007) tested 211 specimens of Scots pine using the Mechanical Timber Grader (MTG), finding that $73 \%$ of the specimens were undergraded and $7 \%$ were overgraded. Íñiguez-González (2007) applied the PLG to large cross-timber of radiata, Scots and Salzmann pine, obtaining similar $\mathrm{R}^{2}$ for MOE estimation from vibration and ultrasound velocity. Santaclara et al. (2009) tested 200 sawn timber pieces of Douglas fir containing a large amount of juvenile wood using PLG, and they found a better $\mathrm{R}^{2}$ in MOE estimation which combined velocity and knottiness parameters rather than velocity and density. Villanueva (2009) tested Spanish juniper round wood by longitudinal vibration, obtaining a $\mathrm{R}^{2}$ of $43 \%$ when estimating MOE by combining Edyn and conicity parameters. Rojas et al. (2011) used a microphone to record the natural frequencies of veneer samples for species identification. Santaclara and Merlo (2011) used the Hitman Director HM200 (HM200) on 162 logs of maritime pine before testing sawn timber from them. A R $\mathrm{R}^{2}$ of $73 \%$ was reported when estimating sawn timber MOE from logs using the Edyn. Arriaga et al. (2012) published the preliminary grading settings for European standard EN 14081-2 (2010) of PLG for Spanish radiata, Scots and Salzmann pine, but were not implemented in the Spanish industry. Montero (2013) tested Scots pine sawn timber with several NDT devices, concluding that PLG results are the best mechanical property estimators. Vega (2013) compared sweet chestnut results from two different vibration devices, the PLG with a microphone and the HM200 with a contact accelerometer, finding better mechanical properties estimation with the PLG measurements. Arriaga et al. (2014) estimated radiata pine mechanical properties based on longitudinal and transversal vibration with similar accuracy. Llana (2016) used the PLG with a microphone and the MTG with a contact accelerometer to estimate MOE with a $91 \% \mathrm{R}^{2}$ and MOR at $70 \%$ using the Edyn, and found no significant differences between the results of both devices. Osuna-Sequera (2017) tested $11 \mathrm{~m}$ long large cross-section Salzmann pine beams from an $18^{\text {th }}$ century timber structure using the PLG and estimating MOE using the Edyn with an $80 \% \mathrm{R}^{2}$. Not only restraint-free isolated specimens were analyzed using the vibration technique, as multiple contact accelerometers were also used to evaluate timber structures. Baño et al. (2011) studied resonance risk in Scots pine timber footbridges, while Castro-Triguero et al. (2017) evaluated a $125 \mathrm{~m}$ length timber footbridge and Arce-Blanco (2017) tested Salzmann pine plank timber arches. Currently, the first research experience on vibration testing of light frame timber floors in Spain is carried out by the Timber Structures and Wood Technology Research Group of the University of Valladolid, after developing their own accelerometers (Villacorta-Calvo et al. 2019). Furthermore, scientists from the previous research group patented a transversal vibration system using several microphone receptors for the evaluation of existing timber structures (Gutiérrez-Sánchez et al. 2019). 


\section{Probing techniques}

Probing methods (needle and drill penetration resistance, screw and nail withdrawal resistance) are mainly used to estimate density in existing timber structures. Palaia et al. (2000) used the Pilodyn to estimate the density of small clear specimens of Scots, maritime and Caribbean pitch pine. Casado et al. (2005) predicted density using the Screw Withdrawal Resistance Meter (SWRM) on 39 Scots pine joists from an existing structure. Bobadilla et al. (2007) estimated density using the Pilodyn and SWRM on 395 large cross-section specimens of radiata, Scots and Salzmann pine with a $\mathrm{R}^{2}$ of $35 \%$ and $49 \%$, respectively. Íñiguez-González et al. (2010) proposed estimation density models for large cross-section radiata, Scots, Salzmann and maritime pine, finding a better $\mathrm{R}^{2}$ with probing techniques than was the case with ultrasound waves. Montón (2012) introduced core drilling technique for density estimation in Spain, obtaining a higher $\mathrm{R}^{2}$ than was the case with the Pilodyn or SWRM in radiata pine. Bobadilla et al. (2013) presented the definitive prototype of the RML Wood Extractor (GICM-UPM, Madrid, Spain) in a NDT wood conference in Madison, WI, USA. The device was designed to be coupled to a commercial drill to collect all of the chips produced during drilling inside a paper bag filter. Density is determined from the mass of chips and the volume of the hole. The UNE 41809 (2014) was published for use of the penetrometer in wood elements to diagnose existing buildings. ÍniguezGonzález et al. (2015a) compared density estimation by using the Pilodyn, SWRM and core drilling, obtaining the highest $\mathrm{R}^{2}$ with the latter. Bobadilla et al. (2018) estimated density by core drilling technique on small clear specimens of 10 species with a $\mathrm{R}^{2}$ of $98 \%$. Llana et al. (2018a) presented a comparison between the Pilodyn, Wood Pecker, SWRM, core drill and RML WoodEx for density estimation of Norway spruce from an existing timber structure, obtaining a better $\mathrm{R}^{2}$ with the core drill and RML WoodEx. The drilling resistance technique using Resistograph and IML Resi devices was used to evaluate timber structures (Capuz et al. 2007, Basterra et al. 2009, Touza 2009, Montoya-Morguí 2010, González-Sanz 2012, Lozano et al. 2013, Abián and Segura 2016) and also for density estimation (Mariño et al. 2002, Casado et al. 2005, Vilches and Correal 2009, Soto-Martínez 2010, Acuña et al. 2011, Morales-Conde et al. 2014, Camacho-Valero 2017).

\section{Other NDT techniques}

Neuronal networks using data from NDT were studied for timber grading (Mier 2001, García-Esteban et al. 2009, García-de-Ceca et al. 2013, García-Iruela et al. 2016, Villasante et al. 2019). Mariño et al. (2010) studied the influence of pith distance on velocity using acoustic tomography. Rodríguez-Abad et al. (2011) used ground-penetrating radar (GPR) on 22 maritime pine joists to estimate MC and Martínez-Sala et al. (2013) studied the differences between longitudinal and transversal GPR measurements. Morales-Conde et al. (2013) used infrared thermography (IRT) to detect MC differences. Oliver and Abián (2013) developed a sensor to monitor timber structures for termites using light emission and fungi risk by moisture content estimation. Sánchez-Beitia et al. (2015) presented the application of Hole-Drilling technique on small clear specimens of radiata pine for stress quantification, and Crespo-de-Antonio et al. (2016) used it to assess two existing timber structures. López et al. (2018) estimated wood density from the variation of surface temperature when specimens are cooled using IRT. Ruano et al. (2019) determined the ratio of juvenile wood to mature wood using near infrared-hyperspectral imaging.

\section{Adjustment factors}

The results of NDT are affected by several factors: moisture content (MC), temperature (T), specimen dimensions, sensor positioning and grain angle and timber-sensor coupling, to mention just a few. Íñiguez-González et al. (2015b) published a compilation of NDT adjustment factors from the national and international literature. Rodríguez-Liñán and Rubio (1995), Rodríguez-Liñán and Rubio (2000) and Palaia et al. (2000) published some of the first Spanish studies of MC influence on NDT measurements using the BPV. Regarding T, Llana et al. (2014) reported the influence of T on NDT, showing a clear linear tendency below $0^{\circ} \mathrm{C}$ and no significant tendency above $0^{\circ} \mathrm{C}$ for dry Scots pine small clear specimens. The length effect was found several times in ultrasound velocity using the SylDuo (Arriaga et al. 2006, Acuña et al. 2007, ÍniguezGonzález et al. 2007a, Llana et al. 2013) and an adjustment procedure was proposed (Llana et al. 2016). The influence of dimension was also reported on the velocity obtained by vibration using PLG (Carballo et al. 2007, Casado et al. 2010). Concerning sensor position with respect to the grain, Rodríguez-Liñán and Rubio (1995) observed a ratio between face to face and end to end velocity $\left(\mathrm{V}_{\mathrm{f}} / \mathrm{V}_{0}\right)$ of 1,19 and between perpendicular and longitudinal velocity $\left(\mathrm{V}_{90} / \mathrm{V}_{0}\right)$ of 2,9. Esteban (2003) found $\mathrm{a}_{90} / \mathrm{V}_{0}$ of 4 and Íñiguez-González et al. (2009) found this to stand at from 2,5 to 3. Several authors proposed adjustments depending on the angle respect to the grain (Acuña et al. 2007, Arriaga et al. 2009, Balmori et al. 2016). Arriaga et al. (2017a) found differences between the velocity obtained in end-to-end measurements and surface or crossed measurements equal to or less than $4,4 \%$ on average. 


\section{Visual grading}

Visual grading is the oldest nondestructive timber evaluation technique. The first Spanish visual grading standard UNE 56525 (1972) was published in December 1972 for structural timber. Seven visual grades were defined (Extra/100, I/80, II/70, III/60, IV/50, V/40 y VI). Argüelles and Arriaga (1986) published a visual grading proposal based on a British standard, with four visual grades for sawn timber $(75,65,50$ and 40) and three for glulam lamellas (LA, LB and LC). A new visual grading standard UNE 56544 (1997) was first published in 1997, first covering softwood and hardwood species (radiata, Scots and maritime pine, black poplar and southern blue gum). Two years later Salzmann pine was also included and afterwards black poplar was excluded. In 2007 a specific standard only for hardwoods was published as UNE 56546 (2007), and the current version of this standard from 2013 is applied to southern blue gum and sweet chestnut, while UNE 56544 (2011) is now only for softwoods. Furthermore, UNE 56547 (2018) is a visual grading standard for Scots and Salzmann pinewood overhead poles. Nowadays national visual grading standards should follow the minimum requirements established by European standard EN 14081-1 (2016). Furthermore, in order to homogenize the national visual grades in all European countries, EN 1912 (2012) related national visual grades with the strength classes according to EN 338 (2016). Concentrated Knot Diameter Ratio (CKDR) is a visual parameter used frequently in combination with NDT ultrasound and vibration results. CKDR includes the influence of knots as the main defect in prediction models, improving the estimation of MOR. This parameter has often been used in Spanish research works, mainly for the assessment of existing structures.

Furthermore, the first research experience of NDT evaluation (using most of the techniques previously cited) of recovered wood from deconstruction and demolition for reuse and recycling purposes is being gained by the Timber Construction Research Group of the Universidad Politécnica de Madrid (Íñiguez-González et al. 2019).

The main goal of this paper is to present the history of wood NDT used in Spain and its main milestones with three objectives. (1) To allow different Spanish and international research groups to have a better knowledge of these works. (2) To elucidate concepts to unify NDT used on timber and future standardization procedures. (3) To promote research group cooperation and exchange activities.

\section{Summary of species and devices used in the literature}

\section{Species}

Structural sawn timber, round wood and small clear specimens from several Spanish-grown species tested with NDT methods were found in the literature: radiata pine (Pinus radiata D. Don), Scots pine (Pinus sylvestris L.), Salzmann pine (Pinus nigra Arnold ssp. salzmannii (Dunal) Franco), Corsican pine (Pinus nigra Arnold ssp. laricio (Poir.) Maire), maritime pine (Pinus pinaster Ait. ssp. mesogeensis Fieschi \& Gaussen and Pinus pinaster Ait. ssp. atlantica H. de Vill.), Aleppo pine (Pinus halepensis Mill.), black poplar (Populus $x$ euramericana (Dode) Guinier), southern blue gum (Eucalyptus globulus Labill.), sweet chestnut (Castanea sativa Mill.), Douglas fir (Pseudotsuga menziesii (Mirb.) Franco), silver fir (Abies alba Mill.), robinia (Robinia pseudoacacia L.), Japanese larch (Larix kaempferi (Lamb.) Carr.), Spanish juniper (Juniperus thurifera L.), European beech (Fagus sylvatica L.), European oak (Quercus robur L.) and Paulownia (Paulownia elongata S.Y.Hu). Some other abroad-grown species but sawn and commercialized in Spain for structural purposes were also found in the literature: missanda (Erythrophleum ivorense A. Chev. and Erythrophleum suaveolens (Guill. \& Perr.) Brenan), iroko (Milicia excelsa (Welw.) C. C. Berg and Milicia regia (A. Chev.) C. C. Berg), Norway spruce (Picea abies (L.) Karst.), southern pine (Pinus tadea L.), American pitch pine (Pinus pallustris Mill.), Caribbean pitch pine (Pinus caribaea Morelet) and western red cedar (Thuja plicata Donn.).

The NDT devices used in Spain are usually portable, and the most common ones cited in the literature are:

\section{Ultrasound and stress wave devices}

Ultrasound and stress wave time-of-flight (ToF) is recorded. The most common devices are: (1) The Steinkamp BP-V (Ultratest, Achim, Germany) ultrasound device (600 V output power) equipped with $50 \mathrm{kHz}$ exponential tip sensors (Figure 2a), (2) The Sylvatest Duo (220-250 V output power) and the Trio (CBS-CBT, Lausanne, Switzerland) instrument equipped with conical $22 \mathrm{kHz}$ sensors (Figure 2b, Figure 2c), (3) The USLab (Agricef, Campinas, Brazil) ultrasound device (700 V output power and 0,1 $\mu$ s resolution) which can be used with different sensors from 20 to $90 \mathrm{kHz}$ (Figure 2e), (4) The MicroSecond Timer (Fakopp, Sopron, Hungary) an impact stress wave device (Figure 2d). Velocity is calculated by dividing length over ToF. 


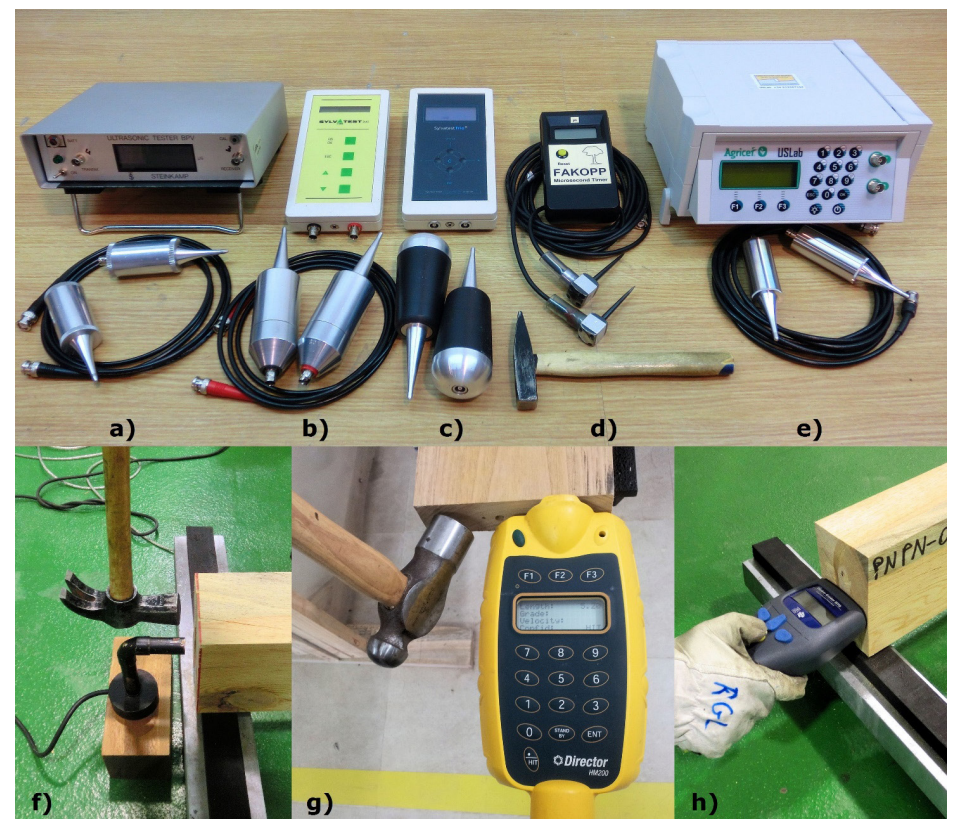

Figure 2: NDT devices: a) Steinkamp BP-V. b) Sylvatest Duo. c) Sylvatest Trio. d) MicroSecond Timer. e) USLab. f) PLG. g) Hitman HM 200 (courtesy of Dr. Abel Vega). h) MTG.

\section{Vibration devices}

Natural frequency data is recorded after inducing vibration by hammer impact. The most common devices found in the literature are: (1) The Portable Lumber Grader PLG (Fakopp, Sopron, Hungary) equipped with a microphone that is placed in front of one end (Figure 2f), (2) The Hitman Director HM 200 (Fibre-gen, Christchurch, New Zealand) equipped with a contact accelerometer (Figure 2g), (3) The Mechanical Timber Grader MTG 960 (Brookhuis, Enschede, Netherlands) equipped with a contact accelerometer (Figure 2h). Velocity from the first mode of natural frequency is calculated as the product of two times length and frequency.

\section{Probing devices}

The most common probing devices used in Spain for density estimation and structural inspections found in the literature review are: (1) The Pilodyn 6 J Forest (Proceq, Schwerzenbach, Switzerland) (Figure 3a). This consists of a calibrated spring that releases a $2,5 \mathrm{~mm}$ diameter steel needle with a constant energy of $6 \mathrm{~J}$. NPR depth of this needle into the timber is measured in mm. (2) The Wood Pecker (DRC, Ancona, Italy) (Figure $3 b$ ). This modified sclerometer inserts a $2,5 \mathrm{~mm}$ diameter steel needle by striking several times with constant energy. NPR depth is measured in mm after each strike. (3) The Screw Withdrawal Resistance Meter SWRM (Fakopp, Sopron, Hungary) (Figure 3c). SWR force is measured in $\mathrm{kN}$ when a standard screw is pulled out. (4) Commercial core bits with different external diameters, usually from 10 to $22 \mathrm{~mm}$ (Figure $3 \mathrm{~d}$ ). The mass and volume of the cylindrical extracted core are measured. (5) The RML Wood Extractor (RML WoodEx) (GICM-UPM, Madrid, Spain) coupled to a commercial drill (Figure 3e). This Spanish design was patented in 2013 (Martínez and Bobadilla 2013) using drilling chips extraction technique. A bit is drilled to a standard depth in wood specimens (so the hollow volume is known) vacuum collecting all of the chips produced during drilling in a paper filter bag. Density is estimated from the mass of chips and volume of the hollow. (6) The Resistograph (RinnTech, Heidelberg, Germany) is a drilling resistance tool where relative resistance is measured against the introduction of a small diameter drill at a constant speed (Figure 3f). (7) The IML Resi (IML, Wiesloch, Germany) uses drilling resistance technique in a similar way to the Resistograph. There are several models, and Figure 3g shows the F400-S. Probing measurements should be taken while avoiding areas close to the pith and other singularities such as knots and resin pockets, etc. 


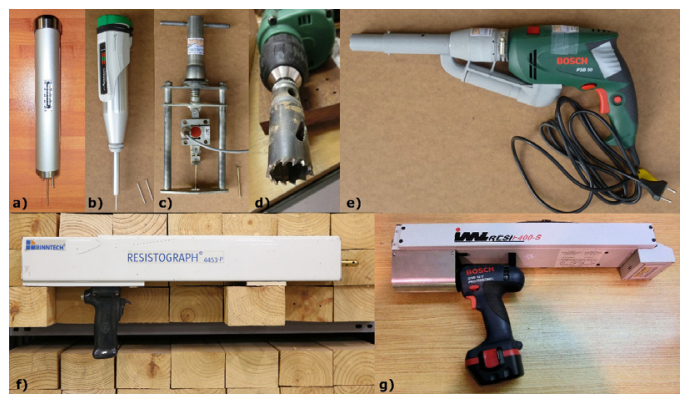

Figure 3: Probing devices: a) Pilodyn 6J Forest. b) Wood Pecker. c) SWRM. d) Core bit. e) RML WoodEx. f) Resistograph (courtesy of Dr. Joaquín Montón). g) IML Resi F400-S.

\section{RESULTS AND DISCUSSION}

\section{Acoustic techniques (ultrasound and stress waves) for property estimation}

Velocity is calculated from ToF by dividing length over ToF. The dynamic modulus of elasticity (Edyn) is calculated as the product of density and square velocity. Several authors have presented mechanical properties estimation models using velocity and Edyn (Table 1).

Table 1: Mechanical properties estimation models by acoustic techniques.

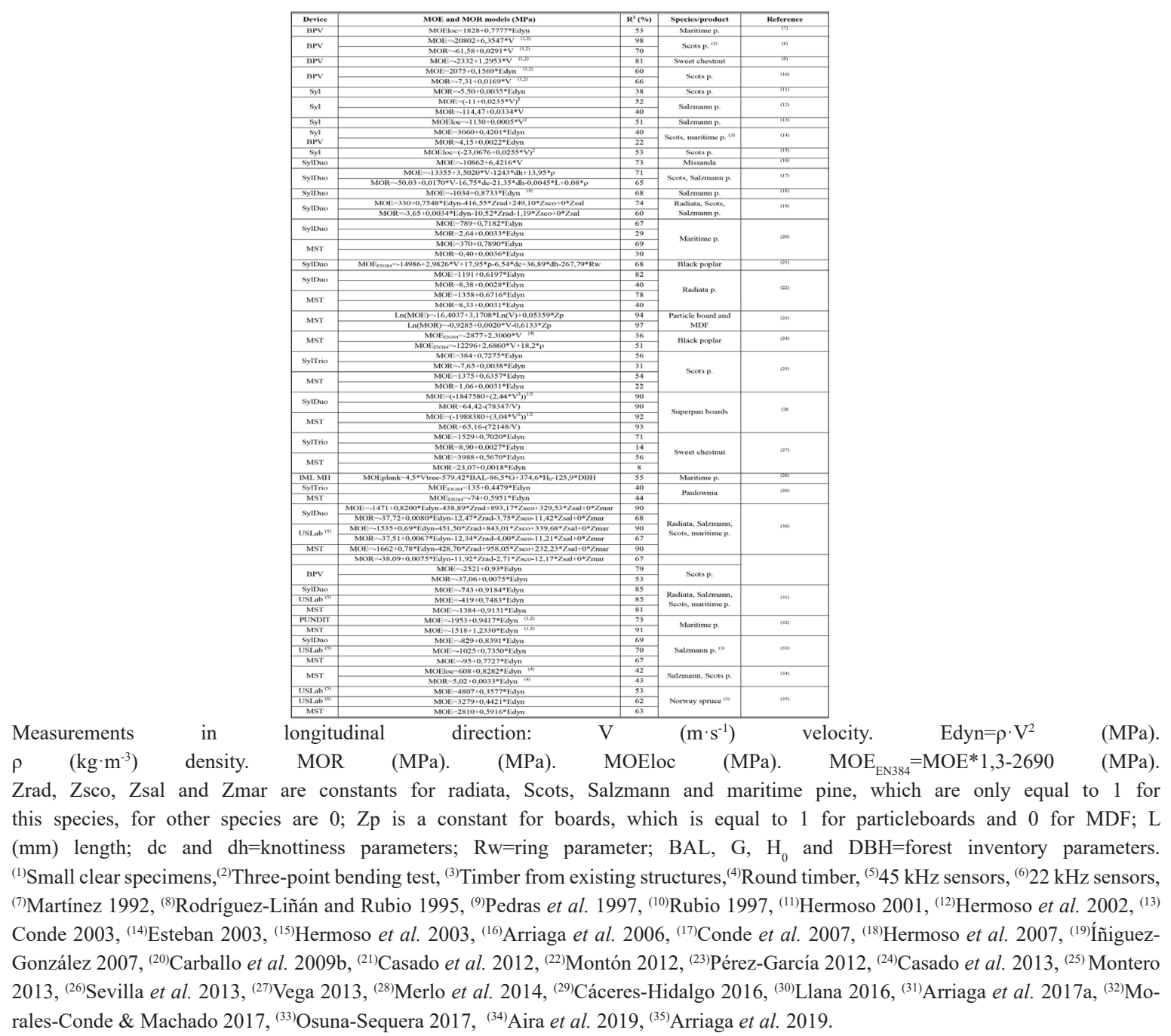




\section{Vibration techniques used to estimate properties}

Longitudinal velocity from first mode natural frequency is calculated as the product of two times length and frequency. The dynamic modulus of elasticity (Edyn) was calculated as product of density and square velocity. Several authors have presented estimation models using vibration techniques (Table 2).

Several authors improved the prediction models of MOR by combining acoustic or vibration results with visual parameters. Hermoso (2001) found an absolute $\mathrm{R}^{2}$ increase of $11 \%$, while the corresponding figure for Íñiguez-González (2007) was $15 \%$ and for Arriaga et al. (2014) it stood at $4 \%$, including knottiness parameters.

Table 2: Mechanical properties estimation models by vibration techniques.

\begin{tabular}{|c|c|c|c|c|}
\hline Device & MOE and MOR models (MPa) & $\mathbf{R}^{2}(\%)$ & Species & Reference \\
\hline \multirow{2}{*}{ PLG } & MOE $=-338+1,1136 * 0,92 *$ Edyn & 77 & \multirow{2}{*}{ Radiata p. } & \multirow{2}{*}{ (3) } \\
\hline & MOR $=0,81+0,0039 *\left(0,92 *\right.$ Edyn- $6,{ }^{*}$ CKDR $)$ & 48 & & \\
\hline \multirow{2}{*}{ MTG } & $\mathrm{MOE}=44,50^{*} \mathrm{Edyn}^{0.6305}$ & 50 & \multirow[b]{2}{*}{ Scots $p$. } & \multirow{2}{*}{ (4) } \\
\hline & MOR $=0,0829 * \mathrm{Edy}^{0.6719}$ & 46 & & \\
\hline \multirow{2}{*}{ PLG } & $\mathrm{MOE}_{\mathrm{EN} 384}=1153+1,04 *\left(0,92^{*} \mathrm{Edyn}-6,2^{*} \mathrm{CKDR}\right)$ & 65 & \multirow{2}{*}{ Scots p. } & \multirow{2}{*}{ (s) } \\
\hline & MOR $=-40,53+0,08 * f+0,07 * \rho$ & 44 & & \\
\hline \multirow{2}{*}{ PLG } & MOE $=762+0,9599 *$ Edyn-508,92*Zrad-354,98Zsco $+0 *$ Zsal & 76 & \multirow{2}{*}{$\begin{array}{l}\text { Radiata, Scots, } \\
\text { Salzmann p. }\end{array}$} & \multirow{2}{*}{ (๑) } \\
\hline & MOR $=3,85+0,0045^{*}$ Edyn- $10,67 *$ Zrad-3,83*Zsco $+0 *$ Zsal & 65 & & \\
\hline \multirow{2}{*}{ PLG } & $\mathrm{MOE}_{\mathrm{EN} 384}=-868+1,0022 *(0,92 *$ Edyn- $6,2 * \mathrm{CKDR})$ & 82 & \multirow{2}{*}{ Maritime p. } & \multirow{2}{*}{ (7) } \\
\hline & MOR $=-70,66+0,0746^{*} \mathrm{f}+0,0641^{*} \rho$ & 47 & & \\
\hline \multirow{2}{*}{ PLG } & $\mathrm{MOE}_{\mathrm{EN} 384}=92+0,7927^{*}\left(0,92^{*} \mathrm{Edyn}\right)$ & 54 & \multirow{2}{*}{ Black poplar } & \multirow{2}{*}{ (8) } \\
\hline & MOR $=-13,08+0,0066^{*}(0,92 *$ Edyn $)$ & 41 & & \\
\hline \multirow{2}{*}{ PLG } & $\mathrm{MOE}=-13294+12,728 * 0+3,689 * \mathrm{~V}$ & 82 & \multirow{2}{*}{ Douglas fir } & \multirow{2}{*}{ (9) } \\
\hline & $\mathrm{MOR}=-43+0,0172 * \mathrm{~V}$ & 38 & & \\
\hline PLG & MOE $=-490+6,7702 * \mathrm{f}+11,10^{*} \rho-262,72 * \mathrm{C}^{(1)}$ & 43 & Spanish juniper & (10) \\
\hline \multirow{2}{*}{ PLG } & MOE $=1642+0,8251^{*}$ Edyn-1041,87*Zrad-176,01*Zsco $+0 *$ Zsal & 72 & \multirow{2}{*}{$\begin{array}{l}\text { Radiata, Scots, } \\
\text { Salzmann p. }\end{array}$} & \multirow{2}{*}{ (11) } \\
\hline & MOR=1,30+0,0038*Edyn-13,25*Zrad-3,01*Zsco+0*Zsal & 61 & & \\
\hline PLG & $\begin{array}{c}\mathrm{MOE}_{\mathrm{EN} 384}=13704+2,9563^{*} \mathrm{~V}+17,85^{*} \rho+307,72 * \mathrm{dc} \\
+62,86 * \mathrm{dh}-267,59 * \mathrm{Rw}\end{array}$ & 70 & Black poplar & (12) \\
\hline \multirow{2}{*}{ PLG } & MOE $=1538+0,7490 *$ Edyn & 85 & Radiata & (13) \\
\hline & MOR=7,95+0,0035*Edyn & 47 & Radiata p. & \\
\hline PIC & MOE $=(-15+0,0040 * \mathrm{~V}+0,0160 * \rho-0,0010 * \mathrm{~L}) * 1000$ & 74 & Sin thot & (14) \\
\hline PLG & MOR $=50,08+0,0034^{*} \mathrm{Edyn}-22,0590^{*} \mathrm{kh}^{12}-0,0090 * \mathrm{~L}$ & 33 & Sweet chestnut & \\
\hline DLC & MOE $=353+0,8734 *$ Edyn & 63 & & \\
\hline PLG & MOR=-9,37+0,0048*Edyn & 37 & & (15) \\
\hline $\operatorname{PIg}(\mathrm{T})$ & $\mathrm{MOE}=3627+0,5564^{*} \mathrm{Edyn}$ & 45 & Scots p. & \\
\hline PLG & MOR $=10,37+0,0028 *$ Edyn & 26 & & \\
\hline PIC & MOE $=1481+0,8230 * E d y n$ & 78 & & \\
\hline PLU & MOR $=3,59+0,0036^{*}$ Edyn & 20 & S & 16 \\
\hline एМ & MOE $=2494+0,7270^{*} \mathrm{Edyn}$ & 70 & Sweet chestnut & \\
\hline HM200 & MOR $=12,46+0,0028 *$ Edyn & 15 & & \\
\hline PLC & MOE $=1229+0,7566^{*} \mathrm{Edyn}$ & 87 & & \\
\hline PLG & MOR $=7,26+0,0035^{*}$ Edyn & 46 & Det. & $(17)$ \\
\hline $\mathrm{PIG}_{(\mathrm{T})}^{(\mathrm{T})}$ & MOE $=823+0,8112^{*}$ Edyn & 86 & Radiata p. & \\
\hline PLG & $\mathrm{MOR}=3,25+0,0040^{*} \mathrm{E}$ dyn & 50 & & \\
\hline PLG & $\mathrm{MOE}_{\mathrm{EN} 384}=357+0,5222 *(0,92 *$ Edyn $)$ & 42 & Paulownia & (18) \\
\hline $\mathrm{PLG}^{(\mathrm{TC})}$ & $\mathrm{MOE}_{\mathrm{EN} 384}=2436+0,9053 * \mathrm{Edyn}$ & 41 & Paulownia & \\
\hline PLG & $\begin{array}{l}\text { MOE }=-226+0,90^{*} \text { Edyn- } 144,26 * \text { Zrad }+ \\
1105,25 * Z s c o+814,85^{*} Z \mathrm{Zsal}+0^{*} \mathrm{Zmar}\end{array}$ & 92 & & \\
\hline & MOR $=-25,99+0,0087 *$ Edyn- $-9,72 *$ Zrad-1,80*Zsco-6,72*Zsal $+0 *$ Zmar & 70 & Radiata, Salzmann, & (19) \\
\hline & MOE $=-327+0,90 *$ Edyn-139,37*Zrad & 92 & Scots, maritime p. & \\
\hline MTG & $\frac{1119,60 * \text { Zsco }+782,94 * \text { Zsal }+0 * \text { Zmar }}{\text { MOR }=-26,92+0,0088 * \text { Edyn- } 9,66 * \text { rad-1,65*Zsco-7,03*Zsal }+0 * \text { mar }}$ & 70 & & \\
\hline PLG & $\mathrm{MOE}=-740+0,9507 *$ Edyn & 80 & Salzmann $\mathrm{p}^{(2)}$ & (20) \\
\hline 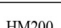 & $\mathrm{MOE}_{\mathrm{EN} 344}=-5353+3,4 * \mathrm{~V}$ & 50 & Radiata p. & (21) \\
\hline HM200 & $\mathrm{MOE}_{\mathrm{EN} 34}=2160+2 * \mathrm{~V}$ & 43 & Maritime p. & \\
\hline
\end{tabular}

Measurements in longitudinal or transversal (T) direction: $\mathrm{V} \quad\left(\mathrm{m} \cdot \mathrm{s}^{-1}\right)$ velocity. $\mathrm{Edyn}=\rho \cdot \mathrm{V}^{2} \quad(\mathrm{MPa})$. $\rho\left(\mathrm{kg} \cdot \mathrm{m}^{-3}\right)$ density. $\mathrm{f}(\mathrm{Hz})$ frequency. MOR (MPa). MOE (MPa). MOEloc (MPa). MOE $\mathrm{EN} 384_{4}=\mathrm{MOE}^{*} 1,3-2690 \quad(\mathrm{MPa})$. Zrad, Zsco, Zsal and Zmar are constants for radiata, Scots, Salzmann and maritime pine, which are only equal to 1 for this species, for other species are 0 ; $\mathrm{L}(\mathrm{mm})$ length; $\mathrm{CKDR}, \mathrm{kh}$, $\mathrm{dc}$ and $\mathrm{dh}=$ knottiness parameters; $\mathrm{C}=$ taper parameter; $\mathrm{Rw}=$ ring parameter. ${ }^{(\mathrm{T})}$ Transversal measurements, ${ }^{(\mathrm{TC})}$ Transversal measurements on cantilever beam, ${ }^{(1)}$ Round timber, ${ }^{(2)}$ Timber from existing structures, ${ }^{(3)} \mathrm{Arria}-$ ga et al. 2005a,${ }^{(4)}$ Broto et al. 2007, ${ }^{(5)}$ Casado et al. 2007, ${ }^{(6)}$ Íñiguez-González 2007, ${ }^{(7)}$ Casado et al. 2008, ${ }^{(8)}$ Casado et al. 2009, ${ }^{\left({ }^{9}\right)}$ Santaclara et al. 2009, ${ }^{(10)}$ Villanueva 2009, ${ }^{(11)}$ Arriaga et al. 2012, ${ }^{(12)}$ Casado et al. 2012, ${ }^{(13)}$ Montón 2012, ${ }^{(14)}$ Vega et al. 2012, ${ }^{(15)}$ Montero 2013, ${ }^{(16)}$ Vega 2013, ${ }^{(17)}$ Arriaga et al. 2014, ${ }^{(18)}$ Cáceres-Hidalgo 2016, ${ }^{(19)}$ Llana 2016, ${ }^{(20)}$ Osuna-Sequera 2017, ${ }^{(21)}$ Vega et al. $2019 \mathrm{~b}$. 


\section{Probing techniques for density estimation}

According to several authors (Bobadilla et al. 2007, Íñiguez-González 2007, Calderón 2012, Martínez 2016) no significant differences were found between radial and tangential measurements (with respect to annual rings). Furthermore, in the assessment of timber structures (the most common use for probing techniques) it is not usually possible to select the probing direction. Density estimation models using acoustic and probing techniques have been presented by several Spanish authors (Table 3).

Table 3: Density estimation models from Spanish research works.

\begin{tabular}{|c|c|c|c|c|c|}
\hline Device & Variable & Density models $\left(\mathrm{kg} \cdot \mathrm{m}^{\mathbf{3}}\right)$ & $\mathbf{R}^{2}(\%)$ & Species/product & Reference \\
\hline $\mathrm{BPV}$ & ToF $(\mu s)$ & $\rho-752,4-0,3201^{*} \mathrm{ToF}$ & 16 & Maritime p. & (1) \\
\hline BPV & Edyn (MPa) & $\rho=634-0,0102^{*} \mathrm{Edyn}$ (II) & 18 & Seots p. & (3) \\
\hline \begin{tabular}{|l} 
Pilodyn \\
\end{tabular} & Depth (mm) & $\rho=711,9-13,9^{*} \mathrm{D}^{(1)}$ & 80 & Scotsp. & (8) \\
\hline IML Resi F300 & Amplitude (\%) & $p^{p=385+21,02 * A^{(1)}}$ & 85 & Scots p. & \\
\hline SWRM & Foree (kN) & $\rho=1000 /(0,956276+(2,3611 / F))$ & 62 & Scots p. ${ }^{2)}$ & (ivi) \\
\hline Resistograph $3450-\mathrm{S}$ & Area $\left(\% \mathrm{~cm}^{-1}\right)$ & $\rho=153+1,51^{*} \mathrm{Ar}$ & 56 & & \\
\hline $\begin{array}{l}\text { Pilodyn } \\
\text { SWRM }\end{array}$ & $\begin{array}{c}\text { Depth }(\mathrm{mm}) \\
\text { Force }(\mathrm{kN})\end{array}$ & $\begin{array}{l}\rho=744,6-22,2^{*} \mathrm{D} \\
\rho=289,9+109,7^{*} \mathrm{~F}\end{array}$ & $\frac{35}{49}$ & Radiata, Scots, Salzmann p. & (11) \\
\hline Pilodyn & Depth (mm) & $\begin{array}{c}\text { p-771,91-19,03*D-97,01*Zrad- } \\
63,19 * \text { Zsco }+0 * \text { Zsal }\end{array}$ & 59 & Radiata, Scols, Salmmann p. & (12) \\
\hline SWRM & Force $(k N)$ & 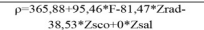 & 64 & & \\
\hline IML Resi F300 & Amplitude & $p_{0=e^{0.13}+\left(0,23^{*} \log A\right)}$ & 68 & Salmmann p. & (13i) \\
\hline SylDuo & \multirow{2}{*}{$\begin{array}{c}\text { Transversal } \\
\text { Velocity }\left(\mathrm{m}^{-} \mathrm{s}^{-1}\right)\end{array}$} & 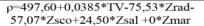 & 34 & \multirow{4}{*}{$\begin{array}{l}\text { Radiata, Scots } \\
\text { Salzmann, maritime p. }\end{array}$} & \multirow{4}{*}{ (a4) } \\
\hline MST & & 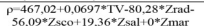 & 34 & & \\
\hline Pilodyn & Depth $(\mathrm{mm})$ & 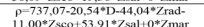 & 61 & & \\
\hline SWRM & Force $(k N)$ & 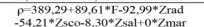 & 67 & & \\
\hline IML Resi E 400 & Amplitude & $\rho=326+19^{*} \mathrm{~A}$ & 44 & Radiata, Scots p. & (15) \\
\hline Resistograph $3450-\mathrm{S}$ & Area $\left(\% \mathrm{~cm}^{-1}\right)$ & $p-394,797+0,7598 * A r$ & 82 & $\begin{array}{l}\text { Salzmann, maritime, Scots p., sweet } \\
\text { chestnut, European oak and walnut }\end{array}$ & (16) \\
\hline SWRM & Force $(\mathrm{kN})$ & $\rho=174,749+64,4308^{*} F$ & 74 & Black poplar & (1) \\
\hline Pilodyn & Depth (mm) & $\rho=700,193-15,9204 * D$ & 31 & \multirow{3}{*}{ Radiata p. } & \multirow{3}{*}{ (ais) } \\
\hline SWRM & Force $(\mathrm{kN})$ & $\rho=285,40+103,77 * \mathrm{~F}$ & 53 & & \\
\hline Core drill bit $囚 16^{(3)}$ & Core $p\left(\mathrm{~kg}^{\prime} \mathrm{m}^{3}\right)$ & $\rho-53,6357+0,850184^{*} \mathrm{CD}$ & 88 & & \\
\hline MST & Velocity $\left(\mathbf{m}^{-} \mathrm{s}^{-1}\right)$ & $\operatorname{Ln}(\rho)=5,42+0,000492^{*} \mathrm{~V}+0,154^{*} Z \mathrm{p}$ & 89 & Particleboard and MDF & (19) \\
\hline Pilodyn & Depth (mm) & $p^{-13,849+5778 / \mathrm{D}}$ & 74 & \multirow{4}{*}{ Scots p. } & (20) \\
\hline Pilodyn & Depth (mm) & $\rho=622,932-11,6226^{*} \mathrm{D}$ & 32 & & \multirow{3}{*}{ (21) } \\
\hline SWRM & Force $(\mathrm{kN})$ & $\rho^{-375,935+85,2801 * \mathrm{~F}}$ & 33 & & \\
\hline Pilodyn + SWRM & $\mathrm{D}(\mathrm{mm})+\mathrm{F}(\mathrm{kN})$ & $\rho-500,663-7,24375 * \mathrm{D}+54,694^{*} \mathrm{~F}$ & 41 & & \\
\hline SylDuo & Velocity $\left(\mathrm{m}^{-} \mathrm{s}^{-1}\right)$ & $\rho=179,65+0,2193^{*} \mathrm{~V}$ & 97 & \multirow{3}{*}{ Superpan boards } & \multirow{3}{*}{ (22) } \\
\hline MST & Velocity $\left(\mathrm{m}^{\prime} \cdot \mathrm{s}^{-1}\right)$ & $\rho=178,39+0,2439 * \mathrm{~V}$ & 98 & & \\
\hline SWRM & Force $(\mathrm{kN})$ & $\rho=341,74+227,458^{*} \mathrm{~F}$ & 90 & & \\
\hline IML Resi-B 1280 & Area/Length (bits) & $\rho=204,4+20,487^{*}$ Ar $/ \mathrm{L}$ & 70 & \multirow{2}{*}{ Pine $^{(2)}$} & \multirow[t]{2}{*}{ (23) } \\
\hline Core drill bit $\emptyset 7^{(3)}$ & Core $\rho\left(\mathrm{kg}^{\prime} \mathrm{m}^{-3}\right)$ & $\rho=122,62+0,6668^{*} \mathrm{CD}(1)$ & 48 & & \\
\hline Pilodyn & Depth $(\mathrm{mm})$ & $\rho=709-15,52 * \mathrm{D}$ & 30 & \multirow{3}{*}{ Radiata p. } & \multirow{3}{*}{ (24) } \\
\hline $\begin{array}{c}\text { SWRM } \\
\text { Core drill bit } \varnothing 10^{(2)}\end{array}$ & Force $(\mathrm{kN})$ & $\rho-294+106,174^{*} \mathrm{~F}$ & 57 & & \\
\hline 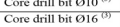 & Core $p\left(\mathrm{~kg}^{\mathrm{m}} \mathrm{m}^{-3}\right)$ & $\frac{\rho-8+0,0,799^{*} \mathrm{CD}}{\rho-87+0.827^{*} \mathrm{CD}}$ & $\frac{80}{80}$ & & \\
\hline Pilodyn & Depth $(\mathrm{mm})$ & 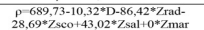 & 56 & \multirow{2}{*}{$\begin{array}{c}\text { Radiata, Scots } \\
\text { Salzmann, maritime p. }\end{array}$} & \multirow{2}{*}{ (25) } \\
\hline SWRM & Force $(\mathrm{kN})$ & 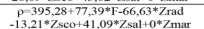 & 68 & & \\
\hline Pilodyn & Depth $(\mathrm{mm})$ & $p=776,09-17,376^{*} \mathrm{D}$ & 51 & \multirow{4}{*}{$\begin{array}{l}\text { Western red cedar, misssanda, black } \\
\text { poplar, sweet chestust, cak, irok, } \\
\text { radiata, Scots, Salzmann, maritime p. } \\
\text { Scots, Salzmann, Alcppo, A. pitch p. }\end{array}$} & \multirow{4}{*}{ (26) } \\
\hline SWRM & Force $(\mathrm{kN})$ & $p^{-445,483+94,63 * F^{(1)}}$ & 53 & & \\
\hline RML WoodEx & Chips Mass (g) & $\rho=-97,59+428,66^{*} \mathrm{ChM}$ (1) & 96 & & \\
\hline IML Resi PD400 & Amplitude (\%) & $p=226,770+8,569^{*} \mathrm{~A}$ & 66 & & \\
\hline IML Resi-B 1280 & Resi $\rho\left(\mathrm{kg} \cdot \mathrm{m}^{3}\right)$ & $\rho=421,9+0,3484^{*} \mathrm{RD} \quad(1)$ & 39 & \multirow{2}{*}{ Maritime $\mathrm{p}$. } & \multirow{2}{*}{ (28) } \\
\hline Core drill bit $\emptyset 7^{(3)}$ & Core $p\left(\mathrm{~kg}^{\prime} \mathrm{m}^{-3}\right)$ & $p=0,9594^{*} \mathrm{CD}$ & 67 & & \\
\hline $\begin{array}{c}\text { Pilodyn } \\
\text { Wood Pecker (") }\end{array}$ & Depth $(\mathrm{mm})$ & 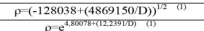 & $\frac{86}{75}$ & Same 10 species Martinez 2016 & (29) \\
\hline Core drill bit $\varrho 10^{(3)}$ & Core Mass (g) & $p_{p-45+228^{\circ} \mathrm{CM}}^{(1)}$ & 98 & Same 10 species Martinez 2016 & (100) \\
\hline $\begin{array}{l}\text { Pilodyn } \\
\text { Wod Porker }\end{array}$ & Depth (mm) & $\mathrm{p}-538-7,25 * \mathrm{D}$ & 22 & & \\
\hline SWRM & Force $(\mathrm{kN})$ & $\frac{\rho p-562-5,70^{*} \mathrm{D}}{\rho=349+64,69^{*} \mathrm{~F}}$ & 53 & & an \\
\hline Core drill bit $凤 10^{(3)}$ & & $p=209+0,47^{4} \mathrm{CD}$ & 84 & Norway spruce ${ }^{(2)}$ & (31) \\
\hline Core drill bit $£ 16^{(0)}$ & Core $p\left(\mathrm{~kg}^{\prime} \mathrm{m}^{-3}\right)$ & $p=270+0,34^{*} \mathrm{CD}$ & 89 & & \\
\hline RML WoodEx & Chips Mass (g) & $\mathrm{p}=195+198,97 * \mathrm{ChM}$ & 70 & & \\
\hline $\begin{array}{c}\text { Infrared } \\
\text { Thermography }\end{array}$ & $\begin{array}{l}\text { T } 10 \min \left({ }^{\circ} \mathrm{C}\right) \\
\text { T30 min }\left({ }^{\circ} \mathrm{C}\right)\end{array}$ & 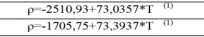 & $\frac{87}{97}$ & $\begin{array}{l}\text { Sapele, moabi, beech, cherry. pine, } \\
\text { oak, ipe }\end{array}$ & (32) \\
\hline BY WOOdEx & Chips Mass (g) & $\rho=-30,79+383,13 * \mathrm{ChM}$ & 84 & Radiata, Scots & (3) \\
\hline & Chips $\rho\left(\mathrm{kg}^{\prime} \mathrm{m}^{-3}\right)$ & $\rho=90,39+0,871 * \mathrm{ChD}$ & 81 & Salmmann, maritime p. & \\
\hline $\begin{array}{l}\text { Pilodyn } \\
\text { (2) }\end{array}$ & Depth $(\mathrm{mm})$ & $\rho=837,41-25,99 * \mathrm{D}$ & 42 & & \\
\hline $\begin{array}{l}\text { Wood Pecker }{ }^{\left({ }^{2}\right)} \\
\text { SWRM }\end{array}$ & Force $(\mathrm{kN})$ & $\frac{p=-974,74-27,76 * \mathrm{D}}{\rho p-316,87+172 * \mathrm{~F}}$ & $\frac{57}{51}$ & Salzmann p. ${ }^{(2)}$ & (34) \\
\hline RML WoodEx & Chips Mass (g) & $\rho-35,94+343,06 * \mathrm{ChM}$ & 76 & & \\
\hline
\end{tabular}

Zrad, Zsco, Zsal and Zmar are the constants for radiata, Scots, Salzmann and maritime pine, which are only equal to 1 for this species, for other species are $0 ; \mathrm{Zp}$ is a constant for boards, which is equal to 1 for particleboards and 0 for MDF, ${ }^{(1)} \mathrm{Small}$ clear specimens, ${ }^{(2)} \mathrm{Timber}$ from existing structures, ${ }^{(3)}$ Internal bit diameter (mm), ${ }^{(4)} 3$ strikes, ${ }^{(5)} 5$ strikes, ${ }^{(6)}$ Martínez 1992, ${ }^{(7)}$ Rubio 1997, ${ }^{(8)}$ Palaia et al. 2000, ${ }^{(9)}$ Mariño et al. 2002, ${ }^{(10)}$ Casado et al. 2005, ${ }^{(1)}$ Bobadilla et al. 2007, ${ }^{(12)}$ Íñiguez-González 2007, ${ }^{(13)}$ Vilches \& Correal 2009, ${ }^{(14)}$ Iñiguez-González et al. 2010, ${ }^{(15)}$ Soto-Martínez 2010, ${ }^{(16)}$ Acuña et al. 2011, ${ }^{(17)}$ Casado et al. 2012, ${ }^{(18)}$ Montón 2012, ${ }^{(19)}$ Pérez-García 2012, ${ }^{(20)}$ Cañas-Gutiérrez 2013, ${ }^{(21)}$ Montero 2013, ${ }^{(22)}$ Sevilla et al. 2013, ${ }^{(23)}$ Morales-Conde et al. 2014, ${ }^{(24)}$ Íñiguez-González et al. 2015a,${ }^{(25)}$ Llana 2016, ${ }^{(26)}$ Martínez 2016, ${ }^{(27)}$ Camacho-Valero 2017, ${ }^{(28)}$ Morales-Conde \& Machado 2017, ${ }^{(29)}$ Salamanca 2017, ${ }^{(30)}$ Bobadilla et al. 2018, ${ }^{\left({ }^{31}\right)}$ Llana et al. 2018a, ${ }^{(32)}$ López et al. 2018, ${ }^{(33)}$ Martínez et al. 2018, ${ }^{(34)}$ Osuna-Sequera et al. 2019b.

\section{MC adjustment factors}

Adjustment factors are important to achieve comparable results. Most research studies focus on MC influence. Palaia et al. (2000) showed that MC influence on ultrasound velocity measured on small clear specimens of Scots, maritime and Caribbean pitch pine varied with a power function. The higher the MC, the lower its influence. Rodríguez-Liñán and Rubio (1995), Llana et al. (2018b) and Llana et al. (2018c) reported 
two different tendencies in which slopes were steeper below fiber saturation point (FSP) than above it, where $\mathrm{MC}$ influence is considered insignificant. Table 4 therefore presents adjustment factors to a reference MC value of $12 \%$, below FSP, as proposed for Spanish-grown species by Equation 1, Equation 2 and Equation 3:

$$
\begin{aligned}
& \operatorname{Vel}_{12 \% M C}=\frac{V e l_{M C}}{\left[1-k_{M C} \times(M C-12)\right]} \\
& \text { Depth }_{12 \% M C}=\frac{\text { Depth }_{M C}}{\left[1+k_{M C} \times(M C-12)\right]} \\
& \text { Force }_{12 \% M C}=\frac{\text { Force }_{M C}}{\left[1-k_{M C} \times(M C-12)\right]}
\end{aligned}
$$

Where: $\operatorname{Vel}_{12 \% \mathrm{MC}}\left(\mathrm{m} \cdot \mathrm{s}^{-1}\right)$ obtained from ToF or longitudinal frequency at $12 \%$ of $\mathrm{MC}, \operatorname{Vel}_{\mathrm{MC}}\left(\mathrm{m} \cdot \mathrm{s}^{-1}\right)$ at a given MC, Depth ${ }_{12 \% \mathrm{MC}}(\mathrm{mm})$ obtained by the Pilodyn $6 \mathrm{~J}$ Forest NPR instrument, Depth ${ }_{\mathrm{MC}}(\mathrm{mm})$ at a given MC, Force $_{12 \% \mathrm{MC}}(\mathrm{kN})$ obtained by the SWRM instrument, Force ${ }_{\mathrm{MC}}(\mathrm{kN})$ at a given MC, $k_{\mathrm{MC}}$ (as per unit) adjustment

\begin{tabular}{|c|c|c|c|c|}
\hline Device & Variable corrected & $k_{\mathrm{MC}}(\%)$ & Species & Reference \\
\hline BPV & Velocity & $0,70^{(1)}$ & Scots $\mathrm{p}$. & (2) \\
\hline Pilodyn & Depth & 1,16 & \multirow{2}{*}{ Radiata p. } & \multirow{2}{*}{ (3) } \\
\hline SWRM & Force & 3,20 & & \\
\hline $\begin{array}{c}\text { PLG } \\
\text { Hitman HM200 }\end{array}$ & Velocity & 1,20 & Sweet chestnut & (4) \\
\hline SylDuo & \multirow{2}{*}{ Velocity } & $0,70^{(1)}$ & \multirow{3}{*}{ Scots p. } & \multirow{3}{*}{ (5) } \\
\hline BPV & & $0,59^{(1)}$ & & \\
\hline Grindosonic MK5 & Edyn & $1,06^{(1)}$ & & \\
\hline SylTrio & \multirow{3}{*}{ Velocity } & 0,48 & \multirow{3}{*}{ Scots p. } & \multirow{3}{*}{ (6) } \\
\hline MST & & 0,50 & & \\
\hline PLG & & 0,65 & & \\
\hline \multirow{4}{*}{$\begin{array}{c}\text { SylDuo } \\
\text { USLab } \\
\text { MST }\end{array}$} & \multirow{4}{*}{ Velocity } & 0,62 & Radiata p. & \multirow{8}{*}{ (7) } \\
\hline & & 0,61 & Scots p. & \\
\hline & & 0,72 & Salzmann $\mathrm{p}$. & \\
\hline & & 0,76 & Maritime $\mathrm{p}$. & \\
\hline \multirow{4}{*}{$\begin{array}{l}\text { PLG } \\
\text { MTG }\end{array}$} & \multirow{4}{*}{ Velocity } & 0,62 & Radiata p. & \\
\hline & & 0,63 & Scots $\mathrm{p}$ & \\
\hline & & 0,73 & Salzmann $\mathrm{p}$. & \\
\hline & & 0,76 & Maritime p. & \\
\hline \multirow{4}{*}{ Pilodyn } & \multirow{4}{*}{ Depth } & 2,20 & Radiata p. & \multirow{8}{*}{ (8) } \\
\hline & & 1,60 & Scots p. & \\
\hline & & 1,70 & Salzmann p. & \\
\hline & & 2,00 & Maritime p. & \\
\hline \multirow{4}{*}{ SWRM } & \multirow{4}{*}{ Force } & 2,20 & Radiata p. & \\
\hline & & 2,80 & Scots p. & \\
\hline & & 2,50 & Salzmann $\mathrm{p}$. & \\
\hline & & 2,10 & Maritime p. & \\
\hline
\end{tabular}
factors, which are listed in Table 4.

Table 4: $\mathrm{MC}$ adjustment factors $\left(k_{\mathrm{MC}}\right)$ in \% for Spanish-grown species (below FSP).

${ }^{(1)}$ Small clear specimens, ${ }^{(2)}$ Rodríguez-Liñán and Rubio 1995, ${ }^{(3)}$ Calderón 2012, ${ }^{(4)}$ Vega 2013, ${ }^{(5)}$ Llana et al. 2014, ${ }^{(6)}$ Montero et al. 2015, ${ }^{(7)}$ Llana et al. 2018b, ${ }^{(8)}$ Llana et al. 2018c. 


\section{Visual grading}

In order to add a new species to the visual grading standard it has to be characterized. Several research works in Spain during the past 30 years focused on this characterization. Fernández-Golfín et al. (1998) summarized the works done in the INIA Structural Timber Laboratory during several years for the characterization of radiata, Scots and maritime pine that led to the production of the first version of the UNE 56544 (1997) standard with two visual grades (ME-1, ME-2). Fernández-Golfín et al. (2001) published the works involved in adding Salzmann pine in the same standard. The results from Íñiguez-González et al. (2007b) made it possible to introduce the new visual grade MEG in the UNE 56544 (2007) for large cross-section timber (thickness > $70 \mathrm{~mm}$ ). Fernández-Golfín et al. (2007) characterized southern blue gum for the first version of the hardwoods visual standard UNE 56546 (2007). Correal et al. (2013) and Vega et al. (2013) proposed visual grading criteria for structural sweet chestnut that were included in UNE 56546 (2013). Preliminary characterization works were also performed for other species that were not included in standards, such as Spanish juniper (Díez et al. 2006). Furthermore, five Spanish species appear in the EN 1912 (2012), and another one has been approved (Table 5). The latest allocations in EN 1912 (2012) were approved according to the works of Vega et al. (2013) and Hermoso et al. (2016). A new revision of the Spanish visual grading standards would be recommendable following the works of Montón et al. (2015), Llana et al. (2019) and the new version of European standard EN 14081:1 (2016). Several research studies were published comparing visual grading according to the Spanish standard UNE 56544 and the German standard DIN 4074-1, (Díez et al. 2000, Conde 2003, Arriaga et al. 2005b, Adell et al. 2008, Llana et al. 2019). In general, more pieces are rejected using the Spanish standard based on knot evaluation. A research work into the load carrying capacity of timber pieces from existing structures (Arriaga et al. 2005b) proposed a visual grading procedure limited to the main parameters (knots and slope of grain) in an attempt to simplify and adapt the procedure used in new timber to in-situ grading particularities. Other works studied the practically zero influence of some defects, such as fissures and wanes, on mechanical properties (Arriaga et al. 2007, Esteban et al. 2010). Touza et al. (2013) proposed a new visual grading criterion for large cross-section American pitch pine specimens from existing structures, based on knots, grain slope and boring insect attacks. Arriaga et al. (2017b) showed that visual grading standards (designed for new sawn timber) lead to a high percentage of rejection in existing timber structures, and it is usually not possible to access all 4 faces. Furthermore, beam cross-section is not homogeneous (Osuna-Sequera et al. 2017). Vega et al. (2019a) found ineffective visual strength grading of 216 dry sweet chestnut small-diameter logs using EN 1927-1 (2008), EN 1927-2 (2008) and DIN 4074-2 (1958) standards.

Table 5: Correspondence between Spanish visual grades and strength classes according to the European standard EN 1912 (2012) and later approvals.

\begin{tabular}{|c|c|c|c|c|c|c|}
\hline \multirow{3}{*}{ Species } & \multirow{3}{*}{ (2) } & \multicolumn{5}{|c|}{ Spanish visual grade } \\
\hline & & \multicolumn{3}{|c|}{ (3) } & \multicolumn{2}{|c|}{ (4) } \\
\hline & & ME1 & ME2 & MEG & MEF & MEF-G \\
\hline Salzmann pine & \multirow{6}{*}{ Strength class } & $\mathrm{C} 30$ & $\mathrm{C} 18$ & $\mathrm{C} 22$ & & \\
\hline Scots pine & & $\mathrm{C} 27$ & $\mathrm{C} 18$ & $\mathrm{C} 22$ & & \\
\hline Radiata pine & & C24 & C18 & $\mathrm{C} 20^{(1)}$ & & \\
\hline Maritime pine & & $\mathrm{C} 24$ & $\mathrm{C} 18$ & - & & \\
\hline Southern blue gum & & & & & D40 & - \\
\hline Sweet chestnut & & & & & D27 $7^{(1)}$ & D24 ${ }^{(1)}$ \\
\hline
\end{tabular}

\footnotetext{
${ }^{(1)}$ Approved by CEN/TC124/WG2-TG1 in October 2014 and not yet included in ${ }^{(2)}$ EN $1912(2012),{ }^{(3)}$ UNE 56544 (2011), ${ }^{(4)}$ UNE 56546
} (2013).

ME1 and ME2: Madera Estructural de $1^{\mathrm{a}}$ y $2^{\mathrm{a}}$ (structural timber $1^{\text {st }}$ and $2^{\text {nd }}$ quality)

MEG: Madera Estructural Gruesa escuadría (large cross-section structural timber)

MEF: Madera Estructural de Frondosas (hardwood structural timber)

MEF-G: Madera Estructural de Frondosas de Gruesa escuadría (hardwood large cross-section structural timber).

\section{Final discussion}

To summarise, 68 mechanical property estimation models from 29 research works were collected in Table 
1 (acoustic techniques), 43 estimation models from 19 research works were included in Table 2 (vibration techniques) and 60 density estimation models from 29 research works were compiled in Table 3 (acoustic and probing techniques). These estimation models were developed from 1992 to 2019 in Spain. Most of these estimation models are valid for the same species, e.g. 24 different models to estimate MOE of the Scots pine (Spanish reference wood species) from ultrasound, stress waves and vibration devices are presented. If these different models are used to calculate MOE from common Spanish-grown Scots pine measurement values (acoustic velocity $5400 \mathrm{~m} \cdot \mathrm{s}^{-1}$, vibration velocity $4750 \mathrm{~m} \cdot \mathrm{s}^{-1}$ and density $510 \mathrm{~kg} \cdot \mathrm{m}^{-3}$, values from Llana (2016)), the mean MOE value obtained is $11734 \mathrm{MPa}$ with a coefficient of variation of $12,6 \%$ and standard deviation of $1474 \mathrm{MPa}$. No significant differences between MOE results of acoustic and vibration techniques were found. From the point of view of the authors, the results should be further studied to elucidate whether the recommended mechanical property estimation models for different NDT devices and species should be included in a new standard or at least in a protocol. However, if end-users develop their own models, these can be used instead of the standardized models. Furthermore, several MC adjustment factors for Spanish-grown species are presented in Table 4 that would be also included in a new standard or protocol. NDT measurement procedures should be unified, e.g. Osuna-Sequera et al. (2019b) concluded that in order to increase the accuracy of density estimation using probing techniques, from three to five measurements in at least two different cross-section areas including the middle point are needed. This should be included in UNE 41809 (2014) as a measurement recommendation.

Better knowledge of the research undertaken should help to prevent overlapping between research groups' works and promote cooperation between them. Some research works presented here are almost unknown: e.g. several interesting and useful results were only published as final degree projects. In 2016 a net of Spanish-timber research groups was created under the name LIGNOMAD to find common objectives and promote collaboration. Research groups should identify potential research objectives, find other research groups with similar objectives and apply together for funding. Furthermore, useful information from previous research works compiled in this review paper can be helpful. E.g. a potential new topic is the reuse and recycling of recovered timber. In this review it was reported that at least one research group in Spain is working on this topic, and several estimation models for timber from existing structures were developed and visual grading criteria for timber from existing structures were proposed. Finally, apart from visual grading, NDT techniques are not used by the Spanish industry for grading purposes, while they are commonly used in most European countries. Therefore, closer collaboration between research groups and industry is needed to implement NDT for grading.

\section{Future milestones}

The main milestones that are expected to be achieved in the near future, given that some Spanish research groups are currently working on them, are: (1) a NDT grading standard for new structural sawn timber, (2) further implementation of NDT in Spanish timber industry, (3) assessment protocol for existing timber structures, including special guidelines for visual grading and for NDT use, (4) models for estimating properties in existing timber structures.

\section{CONCLUSIONS}

Most Spanish research works focus on NDT portable devices which can be used both in new sawn and round timber grading and to assess existing structures. These techniques are not used in practice in the Spanish industry for grading. However, they are frequently used to assess timber structures. Several statistical linear models for the estimation of mechanical properties using different NDT devices (68 models based on acoustic techniques, 43 based on vibration and 60 for density estimation) were developed in Spain from 1992 to 2019, most of them for new sawn timber.

The results obtained are very variable because the methods used are not exactly the same (size of the pieces, wood free of defects vs. structural size timber and the arrangement of measuring equipment, etc.). It is therefore difficult to extrapolate the use of a model for general application. It is very important that in the future different research groups use unified procedures (MC adjustment factors, number of measurements and the way to carry out them) to enhance the capacity of these techniques.

Although many research works have been published in Spanish and in Spanish conferences and work- 
shops, fortunately in recent years more research has been published in English and in scientific journals, allowing international dissemination. Some useful research works presented here are almost unknown. Information from previous research works compiled in this review paper should help research groups to identify potential research objectives, find other research groups with similar objectives and avoid overlapping works.

\section{ACKNOWLEDGMENTS}

Ministerio de Economía y Competitividad (Spanish Ministry of Economy and Competitiveness). Projects: BIA 2014-55089-P; BIA 2010-18858; BIA 2006-14272; AGL 2002-00813; PAT 91-0152; PAT 90-0224. CON09-070; CC02-0031; CON03-001; AGF 99-0176; AGL2004-01598; SC00-043; SC96-045-C2-2. VA047A08. 09MRU004CT. PSING-10-11.

The authors would like to thank Dr. Abel Vega from CETEMAS and Dr. Joaquín Montón from UPC for supplying photographs, together with the laboratory technicians whose work is essential in all research.

\section{REFERENCES}

Abián, M.A.; Segura, G. 2016. Evaluación del estado estructural de la madera después de incendios mediante técnicas no destructivas (Assessment of fire-damaged structural timber by non-destructive testing). In Proceedings of the IV Fire Engineering Conference. Valencia, Spain. 4 p.

Acuña, L.; Díez, M.R.; Martín, L.; Casado, M.; Basterra, L.A.; Ramón, G.; Relea, E. 2007. La técnica de transmisión ultrasónica aplicada a la madera estructural (Ultrasonic transmission technique applied to structural lumber). In Proceedings of the $11^{\circ}$ Congreso Español de Ensayos No Destructivos. Gijón, Spain, pp. 91-102.

Acuña, L.; Basterra, L.A.; Casado, M.; López, G.; Ramón, G.; Relea, E.; Martínez, C.; González, A. 2011. Aplicación del resistógrafo a la obtención de la densidad y la diferenciación de especies de madera (Application of resistograph to obtain the density and to differentiate wood species). Mater Construcc 61(303): 451-464. https://doi.org/10.3989/mc.2010.57610.

Adell, F.J.; Hermoso, E.; Arriaga, F.; Richter, C. 2008. Comparison of the Spanish visual strength grading standard for structural sawn timber (UNE 56544) with the German one (DIN 4074) for Scots pine (Pinus sylvestris L.) from Germany. Holz Roh Werkst 66: 253-258. https://doi.org/10.1007/s00107-008-0241-9.

Aira, J.R.; Villanueva, J.L.; Lafuente, E. 2019. Visual and machine grading of small diameter machined round Pinus sylvestris and Pinus nigra subsp. Salzmannii wood from mature Spanish forests. Mater Struct 52: 32. https://doi.org/10.1617/s11527-019-1330-4.

Arce-Blanco, M. 2017. Estudio de las bóvedas encamonadas en Madrid capital y análisis de su comportamiento estructural (Plank timber arches study in Madrid capital and analysis of structural behavior). Ph.D. Thesis, UPM, Madrid, Spain. https://doi.org/10.20868/UPM.thesis.48247.

Argüelles, R.; Arriaga, F. 1986. Norma de cálculo de estructuras de madera. (Timber structures design standard). AITIM: Madrid, Spain. pp. 80-89.

Arriaga, F.; García, L.; Gebremedhin, K.G.; Peraza, F. 1992. Grading and load carrying capacity of old timber beams. In Proceedings of the International Summer Meeting, American Society of Agricultural Engineers. Charlotte, NC, USA. 25 p.

Arriaga, F.; Íñiguez-González, G.; Esteban, M. 2005a. Assessment of strength and stiffness properties using longitudinal stress wave on structural gross cross section timber of radiate pine (Pinus radiata D. Don). In Proceedings of the $14^{\text {th }}$ International Symposium on Nondestructive Testing of Wood. Hannover, Germany. pp. 103-109. 
Arriaga, F.; Esteban, M.; Relea, E. 2005b. Evaluación de la capacidad portante de piezas de gruesa escuadría de madera de conífera en estructuras existentes (Evaluation of the load carrying capacity of large cross section coniferous timber in standing structures). Mater Construcc 55(280): 43-52. https://doi.org/10.3989/ mc.2005.v55.i280.205.

Arriaga, F.; Íñiguez-González, G.; Esteban, M.; Fernández-Golfín, J.I. 2006. Structural Tali timber (Erythrophleum ivorense A. Chev., Erythrophleum suaveolens Brenan.): assessment of strength and stiffness properties using visual and ultrasonic methods. Holz Roh Werkst 64: 357-362. https://doi.org/10.1007/s00107006-0100-5.

Arriaga, F.; Esteban, M.; Argüelles, R.; Bobadilla, I.; Íñiguez-González, G. 2007. Efecto de las gemas en la resistencia a flexión de piezas enterizas de madera (The effect of wanes on the bending strength of solid timber beams). Mater Construcc 57(288): 61-76. http://materconstrucc.revistas.csic.es/index.php/materconstrucc/article/view/65/78.

Arriaga, F.; Íñiguez-González, G.; Esteban, M.; Bobadilla, I. 2009. Proposal of a methodology for the assessment of existing timber structures in Spain. In Proceedings of the $16^{\text {th }}$ International Symposium on Nondestructive Testing of Wood. Beijing, China. pp. 145-151.

Arriaga, F.; Íñiguez-González, G.; Esteban, M.; Divós, F. 2012. Vibration method for grading of large cross-section coniferous timber species. Holzforschung 66: 381-387. https://doi.org/10.1515/hf.2011.167

Arriaga, F.; Montón, J.; Segués, E.; Íñiguez-González, G. 2014. Determination of the mechanical properties of radiata pine timber by means of longitudinal and transverse vibration methods. Holzforschung 68: 299-305. https://doi.org/10.1515/hf-2013-0087.

Arriaga, F.; Llana, D.F.; Esteban, M.; Íñiguez-González, G. 2017a. Influence of length and sensor positioning on acoustic time-of flight (ToF) measurement in structural timber. Holzforschung 71: 713-723. https:// doi.org/10.1515/hf-2016-0214.

Arriaga, F.; Íñiguez-González, G.; Llana, D.F.; Bobadilla, I.; Esteban, M. 2017b. Procedural considerations for the assessment of mechanical properties in existing timber structures. In Proceedings of the $20^{\text {th }}$ International Nondestructive Testing and Evaluation of Wood Symposium. Madison, WI, USA. pp. 204-212.

Arriaga, F.; Montón, J.; Bobadilla, I.; Llana, D.F. 2019. Influence of length on acoustic time-of-flight (ToF) measurement in built-in structures of Norway spruce timber. Holzforschung 73(4): 339-352. https://doi. org/10.1515/hf-2018-0122.

Atienza-Conejo, L. 2012. Diagnosis de estructuras atacadas por insectos xilófagos mediante ultrasonidos usando la técnica de impulso eco (Timber assessment of structures with xylophagous insect attacks by pulse-echo ultrasound technique). Final Project Degree. UPC, Barcelona, Spain. https://upcommons.upc.edu/ handle/2099.1/14443.

Balmori, J.A.; Acuña, L.; Basterra, L.A. 2016. Estudio de la influencia de la dirección de la fibra en la velocidad de propagación de ultrasonidos (Fakopp) en madera estructural de Pinus sylvestris L. y Pinus radiata D. Don. (Grain angle influence on ultrasound velocity (Fakopp) on Pinus sylvestris L. and Pinus radiata D. Don structural timber). In Proceedings of the Congreso Euro-Americano REHABEND 2016. Burgos, Spain. pp. 746-755.

Baño, V.; Santos, J.C.; Vivas, J.; Rodríguez, S.; Vega, A.; Crews, K. 2011. A study of the influence of different types of timber footbridges on the vibrational natural frequency. In Proceedings of the $17^{\text {th }}$ International Nondestructive Testing and Evaluation of Wood Symposium. Sopron, Hungary. pp. 531-537.

Basterra, L.A.; Acuña, L.; Casado, M.; Ramón, G.; López, G. 2009. Diagnóstico y análisis de estructuras de madera mediante técnicas no destructivas: aplicación a la plaza mayor de Chinchón (Madrid) (Diagnosis and assessment of timber structures using nondestructive techniques: application to the Plaza Mayor in Chinchón (Madrid)). Inf Constr 61(516): 21-36. https://doi.org/10.3989/ic.09.016.

Bobadilla, I.; Íñiguez-González, G.; Esteban, M.; Arriaga, F.; Casas, L. 2007. Density estimation by screw withdrawal resistance and probing in structural sawn coniferous timber. In Proceedings of the $15^{\text {th }}$ International Symposium on Nondestructive Testing of Wood. September 10-12. Duluth, MN, USA. pp. 247-251. 
Bobadilla, I.; Martínez, R.D.; Calvo, J.; Arriaga, F.; Íñiguez-González, G. 2013. First steps in wood density estimation using a conventional drill. In Proceedings of the $18^{\text {th }}$ International Nondestructive Testing and Evaluation of Wood Symposium. September 24-27. Madison, WI, USA. pp. 112-118.

Bobadilla, I.; Martínez, R.D.; Esteban, M.; Llana, D.F. 2018. Estimation of wood density by the core drilling technique. Holzforschung 72(12): 1051-1056. https://doi.org/10.1515/hf-2018-0036.

Broto, M.; Villanueva, J.L.; Lafuente, E.; Rodríguez, F. 2007. Evaluación de la resistencia por frecuencia de resonancia. Un caso práctico (Strength evaluation from resonance frequency: a case study). In Proceedings of the III Congreso Iberoamericano de Productos Forestales. Buenos Aires, Argentina. 1p.

Bucur, V.; Navarrete, A.; Troya, M.T.; Sánchez, E.; Garros, S.; Díez, M.R. 1993. Fungi decay in wood by combined nondestructive testings. In Proceedings of the Ultrasonics International 93 Conference. Vienna, Austria. pp. 287-290.

Cáceres-Hidalgo, E. 2016. Caracterización físico-mecánica de la madera de Paulownia elongata (Physico-mechanical characterization of Paulownia elongata timber). Final Project Master Degree. UVa, Palencia, Spain. http://uvadoc.uva.es/handle/10324/18787.

Calderón, L. 2012. Estudio sobre la influencia del contenido de humedad de la madera en ensayos no destructivos para Pinus nigra Arn., Pinus radiata D. Don y Pinus sylvestris L. (Study of wood moisture content influence on nondestructive measurements on Pinus nigra Arn., Pinus radiata D. Don and Pinus sylvestris L.). Final Project Degree. UPM, Madrid, Spain. http://oa.upm.es/14396/.

Camacho-Valero, J. 2017. Evaluación no destructiva de madera antigua y patrimonial usada estructuralmente (Nondestructive evaluation of structural old heritage timber). Final Project Degree. UPV, Valencia, Spain. https://riunet.upv.es/handle/10251/86871.

Cañas-Gutiérrez, I. 2013. Capacidad de predicción del penetrómetro "Pilodyn" en la determinación de la densidad de la madera (Timber density estimation by Pilodyn penetrometer). Final Project Degree. UVa, Palencia, Spain. http://uvadoc.uva.es/handle/10324/4682.

Capuz, R.; Díez, M.R.; Botelho, J.; San-Valero, E. 2007. Aplicación de métodos de ensayo no destructivos a vigas del forjado del consulado del mar de la Lonja de los Mercaderes de Valencia (NDT evaluation of deck beams in "La Lonja de Mercaderes" in Valencia). In Proceedings of the III Congreso Iberoamericano de Productos Forestales. Buenos Aires, Argentina. 6 p.

Carballo, J.; Hermoso, E.; Fernández-Golfín, J.I. 2007. Influencia del tamaño en la predicción del módulo de elasticidad de vigas de madera de Pinus pinaster con técnicas vibratorias (Influence of the size for elasticity modulus prediction using vibration techniques over Pinus pinaster timber). In Proceedings of the II Jornadas de investigación en construcción. Instituto de ciencias de la construcción Eduardo Torroja. Madrid, Spain. 10 p.

Carballo, J.; Hermoso, E.; Díez, M.R. 2009a. Ensayos no destructivos sobre madera estructural. Una revisión de 30 años en España. (Non-destructive testing on structural timber. A review of last 30 years in Spain). Revista forestal (Costa Rica) 6(17): 1-16. https://revistas.tec.ac.cr/index.php/kuru/article/view/387.

Carballo, J.; Hermoso, E.; Fernández-Golfín, J.I. 2009b. Comparación de la evaluación y clasificación mecánica del Pinus pinaster Ait. con dos equipos de ultrasonidos (Comparison of evaluation and mechanical grading of Pinus pinaster Ait. by two ultrasound devices). In Proceedings of the $5^{\circ}$ Congreso Forestal Español. Ávila, Spain. 12 p.

Casado, M.; Basterra, L.A.; Acuña, L.; Pinazo, O.; Martínez, C.; Relea, E.; Barranco, I.; Ramón, G. 2005. Determinación de la capacidad resistente mediante métodos no destructivos. Aplicación en viguetas de forjado de un edificio singular (Determination of resistance by non destructive testing applied to structural timber joists from a singular building). In Proceedings of the IV Congreso Forestal Español. Zaragoza, Spain. 7 p.

Casado, M.; Acuña, L.; Vecilla, D.; Basterra, L.A.; Pando, V.; Relea, E. 2007. Determinación de la capacidad resistente de madera estructural de Pinus sylvestris mediante PLG (Determination of bending strength of structural timber of Pinus sylvestris by PLG). In Proceedings of the $11^{\circ}$ Congreso Español de Ensayos No Destructivos. Gijón, Spain. 10 p. 
Casado, M.; Acuña, L.; Basterra, L.A.; Relea, E. 2008. Clasificación de madera estructural de Pinus pinaster mediante técnicas vibratorias (Grading of Pinus pinaster structural timber by vibration techniques). In Proceedings of the II Jornadas de Investigación en Construcción. Instituto de Ciencias de la Construcción Eduardo Torroja. Madrid, Spain. 9 p.

Casado, M.; Escudero, I.; Acuña, L.; Vecilla, D.; Basterra, L.A.; Ramón, G.; López, G.; Relea, E. 2009. Técnicas vibratorias aplicadas a madera estructural de Populus x euramericana (Application of vibration techniques to structural timber of Populus $x$ euramericana). In Proceedings of the $5^{\circ}$ Congreso Forestal Español. Avila, Spain. 14 p.

Casado, M.; Acuña, L.; Vecilla, D.; Relea, E.; Basterra, L.A.; Ramón, G.; López, G. 2010. The influence of size in predicting the elastic modulus of Populus $x$ euramericana timber using vibration techniques. In Structures and Architecture. Taylor \& Francis Group, London, UK. pp. 2025-2032. http://maderas.uva.es/ files/2019/03/2010-Casado-et-al-The-influence-of-size-MOE-Populus-PLG.pdf.

Casado, M.; Acuña, L.; Basterra, L.A.; Ramón, G.; Vecilla, D. 2012. Grading of structural timber of Populus x euroamericana clone I-214. Holzforschung 66(5): 633-638. https://doi.org/10.1515/hf-2011-0153.

Casado, M.; Acuña, L.; Basterra, L.A.; Heredero, S.; SanMartín, R. 2013. Estimación de la calidad de la madera en rollo de Populus x euramericana mediante ultrasonidos (Estimation of round timber quality of Populus $x$ euramericana by ultrasound). In Proceedings of the $6^{\circ}$ Congreso Forestal Español. Vitoria, Spain. $11 \mathrm{p}$.

Castro-Triguero, R.; García-Macías, E.; Saavedra-Flores, E.; Friswell, M.I.; Gallego, R. 2017. Multi-scale model updating of a timber footbridge using experimental vibration data. Eng Computation 34(3): 754-780. https://doi.org/10.1108/EC-09-2015-0284.

Conde, M. 2003. Caracterización de la madera estructural de Pinus nigra Subsp. Salzmannii. (Characterization of structural timber from Pinus nigra Ssp. Salzmannii). Ph.D. Thesis, UPM, Madrid, Spain.

Conde, M.; Fernández-Golfín, J.I.; Hermoso, E. 2007. Mejora de la predicción de la resistencia y rigidez de la madera estructural con el método de ultrasonidos combinado con parámetros de clasificación visual (Improving the prediction of strength and rigidity of structural timber by combining ultrasound techniques with visual grading parameters). Mater Construcc 57(288): 49-59. http://materconstrucc.revistas.csic.es/index. $\mathrm{php} /$ materconstrucc/article/view/64/77.

Correal, E.; Vilches, M.; Iglesias, C. 2013. Clasificación visual de la madera estructural de Castanea sativa del Sistema Mediterráneo Catalán (Visual grading for structural sawn timber of Castanea sativa from Catalan Mediterranean source). In Proceedings of the $6^{\circ}$ Congreso Forestal Español. Vitoria, Spain. $12 p$.

Crespo, J.; Aira, J.R.; Vázquez, C.; Guaita, M. 2017. Comparative analysis of the elastic constants measured via conventional, ultrasound, and 3-D digital image correlation methods in Eucalyptus globulus Labill. BioResources 12(2): 3728-3743. https://doi.org/10.15376/biores.12.2.3728-3743.

Crespo-de-Antonio, M.; Luengas-Carreño, D.; Sánchez-Beitia, S. 2016. Applications of the hole-drilling technique in timber structures. Case studies. In Proceedings of the $10^{\text {th }}$ International Conference on Structural Analysis of Historical Constructions. Leuven, Belgium. pp. 776-781.

Díez, M.R.; Conde, M.; Fernández-Gofín, J.I.; Rosskopf, S. 2000. Clasificación visual de madera estructural de pino laricio (Pinus nigra Arn.): Comparación de resultados usando las normas UNE 56544 y DIN 4074 (Visual grading of structural timber of Salzmann pine (Pinus nigra Arn.): Comparison of results using standards UNE 56544 and DIN 4074). Inv Agrar-Sist Rec F 9(2): 375-380. https://recyt.fecyt.es/index. php/IA/article/view/2630/2007.

Díez, M.R.; Cabrero-Rojo, J.C.; García-Lombardero, R. 2006. Caracterización de la sabina albar con piezas de tamaño casi estructural. Intentos preliminares (Spanish juniper characterization of almost structural size timber. Preliminary results). In Proceedings of the III Coloquio Internacional sobre Sabinares y Enebrales (género Juniperus): Ecología y gestión forestal sostenible. Soria, Spain. 5 p.

DIN. 1958. Bauholz für Holzbauteile; Gütebedingungen für Baurundholz (Nadelholz) (Building Timber for Wood Building Components; Quality Conditions for Building Logs (Softwood)). DIN 4074-2. 1958. Nor- 
menausschuss Holzwirtschaft und Möbel (NHM): Berlin, Germany.

DIN. 2012. Sortierung von Holz nach der Tragfähigkeit - Teil 1: Nadelschnittholz (Strength grading of wood - Part 1: Coniferous sawn timber). DIN 4074-1. 2012. Normenausschuss Holzwirtschaft und Möbel (NHM): Berlin, Germany.

Esteban, M. 2003. Determinación de la capacidad resistente de la madera estructural de gran escuadría y su aplicación en estructuras existentes de madera de conífera (Determination of the load carrying capacity of large cross-section structural coniferous timber in existing structures). Ph.D. Thesis, UPM, Madrid, Spain. http://oa.upm.es/1404/.

Esteban, M.; Bobadilla, I.; Arriaga, F.; Íñiguez-González, G.; García, H. 2009. NDT applied to estimate the mechanical properties of the timber of an ancient structure in Valsaín, Segovia (Spain). In Proceedings of the $16^{\text {th }}$ International Symposium on Nondestructive Testing of Wood. Beijing, China. pp. 152-157.

Esteban, M.; Arriaga, F.; Íñiguez-González, G.; Bobadilla, I.; Mateo, R. 2010. Influencia de las fendas en la resistencia de la madera estructural (The effect of fissures on the strength of structural timber). Mater Construcc 60(299): 115-132. https://doi.org/10.3989/mc.2010.48208.

European Committee of Standardization. CEN. 2008. Qualitative classification of softwood round timber - Part 1: Spruces and firs. EN 1927-1. 2008. European Committee of Standardization (CEN): Brussels, Belgium.

European Committee of Standardization. CEN. 2008. Qualitative classification of softwood round timber - Part 2: Pines. EN 1927-2. 2008. European Committee of Standardization (CEN): Brussels, Belgium.

European Committee of Standardization. CEN. 2010. Timber structures - Strength graded structural timber with rectangular cross section - Part 2: Machine grading; additional requirements for initial type testing. EN 14081-2. 2010. European Committee of Standardization (CEN): Brussels, Belgium.

European Committee of Standardization. CEN. 2012. Structural timber. Strength classes. Assignment of visual grades and species. EN 1912. 2012. European Committee of Standardization (CEN): Brussels, Belgium.

European Committee of Standardization. CEN. 2016. Structural timber. Strength classes. EN 338. 2016. European Committee of Standardization (CEN): Brussels, Belgium.

European Committee of Standardization. CEN. 2016. Timber structures - Strength graded structural timber with rectangular cross section - Part 1: General requirements. EN 14081-1. 2016. European Committee of Standardization (CEN): Brussels, Belgium.

Fernández-Golfín, J.I.; Díez, M.R.; Gutiérrez-Oliva, A. 1998. Caracterización mecánica de la madera aserrada de uso estructural, clasificada visualmente de acuerdo con la norma UNE56544 (Mechanical characterization of sawn timber for structural use, graded visually in accordance with Spanish standard UNE56544). Mater Construcc 48(252): 45-59. http://materconstrucc.revistas.csic.es/index.php/materconstrucc/article/ view/463/511.

Fernández-Golfín, J.I.; Díez, M.R.; Baonza, M.V.; Gutiérrez-Oliva, A.; Hermoso, E.; Conde, M.; Van den Eynde, V. 2001. Caracterización de la calidad y las propiedades de la madera de Pino laricio (Pinus nigra Arn. salzmannii) (Quality and properties characterization of Salzmann pine (Pinus nigra Arn. salzmannii) timber). Inv Agrar-Sist Rec F 10(2): 311-331. http://www.inia.es/IASPF/2001/vol10-2/ferna.PDF.

Fernández-Golfín, J.I.; Díez, M.R.; Hermoso, E.; Baso, C.; Casas, J.M.; González, O. 2007. Caracterización de la madera de Eucalyptus globulus para uso estructural (Characteristics of eucalyptus wood for structural purposes). CIDEU 4: 91-100. https://dialnet.unirioja.es/descarga/articulo/2723431.pdf.

Galvañ, V.; Palaia, L.; Cervera, F.; Monzo, V. 1994. Strength estimation of decayed timber in old buildings by means of ultrasonic devices. In Proceedings of the $1^{\text {st }}$ European Symposium on Nondestructive Evaluation of Wood. Sopron, Hungary. pp. 156-170.

García-de-Ceca, J.L.; Hermoso, E.; Mateo, R.; Íñiguez-González, G. 2013. Neural network models for 
the establishment of a structural stress grading methodology using nondestructive techniques. In Proceedings of the $18^{\text {th }}$ International Nondestructive Testing and Evaluation of Wood Symposium. Madison, WI, USA. p. 808.

García-Esteban, L.; García-Fernández, F.; Palacios, P. 2009. MOE prediction in Abies pinsapo Boiss. timber: Application of an artificial neural network using non-destructive testing. Comput Struct 87: 1360-1365. https://doi.org/10.1016/j.compstruc.2009.08.010.

García-Iruela, A.; García-Fernández, F.; García-Esteban, L.; Palacios, P.; Simón, C.; Arriaga, F. 2016. Comparison of modelling using regression techniques and an artificial neural network for obtaining the static modulus of elasticity of Pinus radiata D. Don. timber by ultrasound. Compos Part B-Eng 96: 112-118. https://doi.org/10.1016/j.compositesb.2016.04.036.

González-Sanz, M. 2012. Estudio sobre el estado de conservación de la cubierta del martinete de Navafría (Segovia) (Assessment of roof structure of Navafría forge (Segovia)). Final Project Degree. UPM, Madrid, Spain. http://oa.upm.es/36345/.

Gutiérrez-Sánchez, R.; Basterra, L.A.; Acuña, L.; Casado, M.; Morillas, L.; López, G.; Balmori, J.A. 2019. Sistema y procedimiento de medición sónico para diagnóstico de elementos estructurales de madera. WO2018178482. España. https://patentscope.wipo.int/search/es/detail.jsf?docId=WO2018178482.

Hermoso, E. 2001. Caracterización mecánica de la madera estructural de Pinus sylvestris L. (Mechanical characterization of Pinus sylvestris L. structural timber). Ph.D. Thesis, UPM, Madrid, Spain. http://oa.upm. es/644/.

Hermoso, E.; Díez, M.R.; Fernández-Golfín, J.I.; Mier, R. 2002. Calidad de la madera aserrada mediante evaluación no destructiva con ultrasonidos (Quality assessment of sawn timber by ultrasound non-destructive testing). In Proceedings of the II Congreso Nacional de la Madera. Segovia, Spain. 13 p.

Hermoso, E.; Fernández-Golfín, J.I.; Díez, M.R. 2003. Evaluación de la clasificación resistente de la madera estructural mediante ultrasonidos (Evaluation of structural timber strength grading by ultrasound). In Proceedings of the the $10^{\circ}$ Congreso Nacional de Ensayos No Destructivos. Cartagena, Spain. 9 p.

Hermoso, E.; Fernández-Golfín, J.I.; Díez, M.R.; Mier, R. 2007. Aplicación de los ultrasonidos a la evaluación de las propiedades mecánicas de la madera en rollo de pequeño diámetro (Ultrasound application to evaluation of small round timber mechanic properties). Inf Constr 59(506): 87-95. http://informesdelaconstruccion.revistas.csic.es/index.php/informesdelaconstruccion/article/view/511/586.

Hermoso, E.; Mateo, R.; Íñiguez-González, G.; Montón, J.; Arriaga, F. 2016. Visual grading and structural properties assessment of large cross-section Pinus radiata D. Don timber. BioResources 11(2): 53125321. https://doi.org/10.15376/biores.11.2.5312-5321.

Hillig, E.; Bobadilla, I.; Gonçalves, R.; Llana, D.F. 2018. The influence of wood polymer composite (WPC) specimen composition and dimensions on wave propagation. Eur J Wood Prod 76(4): 1153-1164. https://doi.org/10.1007/s00107-018-1309-9.

Íñiguez-González, G. 2007. Clasificación mediante técnicas no destructivas y evaluación de las propiedades mecánicas de la madera aserrada de coníferas de gran escuadría para uso estructural. (Grading by non destructive techniques and assessment of the mechanical properties of large cross section coniferous sawn timber for structural use). Ph.D. Thesis, UPM, Madrid, Spain. http://oa.upm.es/415/.

Íñiguez-González, G.; Esteban, M.; Arriaga, F.; Bobadilla, I.; Gil, M.C. 2007a. Influence of specimen length on ultrasound wave velocity. In Proceedings of the $15^{\text {th }}$ International Symposium on Nondestructive Testing of Wood. Duluth, MN, USA. pp. 155-159.

Íñiguez-González, G.; Arriaga, F.; Barrett, J.D.; Esteban, M. 2007b. Visual grading of large structural coniferous sawn timber according to Spanish standard UNE 56544. Forest Prod J 57(10): 45-50.

Íñiguez-González, G.; Martínez, R.D.; Bobadilla, I.; Arriaga, F.; Esteban, M. 2009. Mechanical properties assessment of structural coniferous timber by means of parallel and perpendicular to the grain wave velocity. In Proceedings of the $16^{\text {th }}$ International Symposium on Nondestructive Testing of Wood. Beijing, 
China. pp. $79-84$.

Íñiguez-González, G.; Arriaga, F.; Esteban, M.; Bobadilla, I.; González, C.; Martínez, R.D. 2010. In situ non-destructive density estimation for the assessment of existing timber structures. In Proceedings of the World Conference on Timber Engineering (WCTE). Riva di Garda, Italy. 8 p.

Íñiguez-González, G.; Montón, J.; Arriaga, F.; Segués, E. 2015a. In-situ assessment of structural timber density using non-destructive and semi-destructive testing. BioResources 10(2): 2256-2265. https://bioresources.cnr.ncsu.edu/resources/in-situ-assessment-of-structural-timber-density-using-non-destructive-and-semi-destructive-testing/.

Íñiguez-González, G.; Arriaga, F.; Esteban, M.; Llana, D.F. 2015b. Reference conditions and modification factors for the standardization of nondestructive variables used in the evaluation of existing structures. Constr Build Mater 101:1166-1171. https://doi.org/10.1016/j.conbuildmat.2015.05.128.

Íñiguez-González, G.; Arriaga, F.; Osuna-Sequera, C.; Esteban, M.; Ridley-Ellis, D. 2019. Nondestructive measurements in reclaimed timber from existing structures. In Proceedings of the $21^{\text {st }}$ International Nondestructive Testing and Evaluation of Wood Symposium. Freiburg im Breisgau, BW, Germany. pp. $462-$ 472 .

Llana, D.F.; Sanabria, S.J.; Íñiguez-González, G.; Arriaga, F.; Niemz, P. 2013. Experimental and numerical investigation of effect of sawn timber dimensions in ultrasonic velocity measurements for Spanish softwoods. In Proceedings of the $18^{\text {th }}$ International Nondestructive Testing and Evaluation of Wood Symposium. Madison, WI, USA. p. 710.

Llana, D.F.; Íñiguez-González, G.; Arriaga, F.; Niemz, P. 2014. Influence of temperature and moisture content on non-destructive measurements in Scots pine wood. Wood Research 59(5): 769-780. http://www. woodresearch.sk/wr/201405/06.pdf.

Llana, D.F. 2016. Influencia de factores físicos y geométricos en la clasificación estructural de la madera mediante técnicas no destructivas (The influence of physical and geometrical factors on timber stress-grading by non-destructive techniques). Ph.D. Thesis, UPM, Madrid, Spain. https://doi.org/10.20868/UPM.thesis. 43696 .

Llana, D.F.; Íñiguez-González, G.; Arriaga, F.; Wang, X. 2016. Time-of-flight adjustment procedure for acoustic measurements in structural timber. BioResources 11(2): 3303-3317. https://doi.org/10.15376/ biores.11.2.3303-3317.

Llana, D.F.; Íñiguez-González, G.; Montón, J.; Arriaga, F. 2018a. In-situ density estimation by four nondestructive techniques on Norway spruce from built-in wood structures. Holzforschung 72(10): 871-879. https://doi.org/10.1515/hf-2018-0027.

Llana, D.F.; Íñiguez-González, G.; Martínez, R.D.; Arriaga, F. 2018b. Influence of timber moisture content on wave time-of-flight and longitudinal natural frequency in coniferous species for different instruments. Holzforschung 72(5): 405-411. https://doi.org/10.1515/hf-2017-0113.

Llana, D.F.; Hermoso, E.; Bobadilla, I.; Íñiguez-González, G. 2018c. Influence of moisture content on the results of penetration and withdrawal resistance measurements on softwoods. Holzforschung 72(7): 549555. https://doi.org/10.1515/hf-2017-0133.

Llana, D.F.; Arriaga, F.; Esteban, M.; Íñiguez-González, G. 2019. Comparison between wet and dry timber visual strength grading according to the Spanish (UNE 56544) and German (DIN 4074-1) standards. Mater Construcc 69(336): e205. https://doi.org/10.3989/mc.2019.03319.

López, G.; Basterra, L.A.; Acuña, L. 2018. Infrared thermography for wood density estimation. Infrared Phys Techn 89: 242-246. https://doi.org/10.1016/j.infrared.2018.01.015.

Lozano, A.; Guaita, M.; Lorenzo, D.; Benito, J. 2013. Investigation about the decay of the timber structure of an ancient wine cellar in La Rioja (Spain) and sanitation proposal. Adv Mater Res-Switz 778: 976-982. https://doi.org/10.4028/www.scientific.net/AMR.778.976. 
Mariño, R.A.; Fernández, M.E.; Fernández-Rodríguez, C. 2002. Análisis comparativo de la densidad de la madera de Pinus sylvestris L. mediante la utilización del resistógrafo (Comparative analysis of Pinus sylvestris L. wood density using resistograph). Revista CIS-Madera 9: 60-70.

Mariño, R.A.; Fernández, M.E.; Fernández-Rodríguez, C.; Méndez, M. 2010. Detection of pith location in chestnut lumber (Castanea sativa Mill.) by means of acoustic tomography and longitudinal stress-wave velocity. Eur J Wood Prod 68: 197-206. https://doi.org/10.1007/s00107-009-0366-5.

Martín, M.T. 1994. Some nondestructive methods to determine physical features of wood. In Proceedings of the $1^{\text {st }}$ European Symposium on Nondestructive Evaluation of Wood. Sopron, Hungary. p. 536.

Martínez, J.J. 1992. Características mecánicas de la madera de Pinus pinaster Ait. obtenidas a partir de ensayos con piezas de tamaño estructural (Mechanical properties of Pinus pinaster Ait. structural timber). Ph.D. Thesis, UPM, Madrid, Spain.

Martínez, R.D.; Bobadilla, I. 2013. Extractor de muestras de madera mediante taladro (Wood sample extractor using a conventional drill). Spain, ES2525504. (B27C 3/00) (2006.1). Appl. 201330890. https:// patentscope.wipo.int/search/es/detail.jsf?docId=ES132634337\&_cid=P22-K403XF-18800-1.

Martínez, R.D. 2016. Métodos no destructivos de estimación de la densidad de madera. (Timber density estimation by non-destructive methods). Ph.D. Thesis. USC, Lugo, Spain. http://hdl.handle.net/10347/15159.

Martínez, R.D.; Calvo, J.; Arriaga, F.; Bobadilla, I. 2018. In situ density estimation of timber pieces by drilling residue analysis. Eur J Wood Prod 76: 509-515. https://doi.org/10.1007/s00107-017-1214-7.

Martínez-Sala, R.; Rodríguez-Abad, I.; Díez, M.R.; Capuz, R. 2013. Assessment of the dielectric anisotropy in timber using the nondestructive GPR technique. Constr Build Mater 38: 903-911. https://doi. org/10.1016/j.conbuildmat.2012.09.052.

Merlo, E.; Álvarez-González, J.G.; Santaclara, O.; Riesco, G. 2014. Modelling modulus of elasticity of Pinus pinaster Ait. in northwestern Spain with standing tree acoustic measurements, tree, stand and site variables. Forest Syst 23(1): 153-166. http://dx.doi.org/10.5424/fs/2014231-04706.

Mier, R. 2001. Clasificación de madera aserrada estructural mediante Inteligencia Artificial: Redes Neuronales (Structural sawn timber classification by artificial intelligent: Neural Networks). Final Project Degree. UPM, Madrid, Spain.

Montero, M.J. 2013. Clasificación de madera estructural de gran escuadría de Pinus sylvestris L. mediante métodos no destructivos (Grading of large cross section structural timber of Pinus sylvestris L. by non destructive techniques). Ph.D. Thesis, UPM, Madrid, Spain. http://oa.upm.es/15201/.

Montero, M.J.; de la Mata, J.; Esteban, M.; Hermoso, E. 2015. Influence of moisture content on the wave velocity to estimate the mechanical properties of large cross-section pieces for structural use of Scots pine from Spain. Maderas-Cienc Tecnol 17(2): 407-420. http://dx.doi.org/10.4067/S0718-221X2015005000038.

Montón, J. 2012. Clasificación estructural de la Madera de Pinus radiata D. Don procedente de Cataluña mediante métodos no destructivos y su aplicabilidad en la diagnosis estructural (Structural timber grading of Pinus radiata D. Don from Catalonia using NDT and applicability in structural assessment). Ph.D. Thesis. UPC. Barcelona, Spain. https://www.tdx.cat/handle/10803/96423\#page=1.

Montón, J.; Arriaga, F.; Íñiguez-González, G.; Segués, E. 2015. Warp requirements and yield efficiency in the visual grading of sawn radiata pine timber. BioResources 10(1): 1115-1126. https://bioresources.cnr. ncsu.edu/resources/warp-requirements-and-yield-efficiency-in-the-visual-grading-of-sawn-radiata-pine-timber/.

Montoya-Morguí, F. 2010. Creació d'una metodologia d'aplicació i assaig del Resistógraf en la diagnosi d'elements estructurals de fusta (Resistograph application and evaluation methodology development for assessment of timber structures). Final Project Degree. UPC, Barcelona, Spain.

Morales-Conde, M.J.; Rodríguez-Liñán, C.; Rubio, P. 2013. Application of non-destructive techniques in the inspection of the wooden roof of historic buildings: A case study. In Proceedings of the $2^{\text {nd }}$ International 
Conference on Structural Health Assessment of Timber Structures (SHATIS). Trento, Italy. pp. 233-242.

Morales-Conde, M.J.; Rodríguez-Liñán, C.; Machado, J.S. 2014. Predicting the density of structural timber members in service. The combined use of wood cores and drill resistance data. Mater Construcc 64(315): e029. http://dx.doi.org/10.3989/mc.2014.03113.

Morales-Conde, M.J.; Machado, J.S. 2017. Evaluation of cross-sectional variation of timber bending modulus of elasticity by stress waves. Constr Build Mater 134: 617-625. https://doi.org/10.1016/j.conbuildmat.2016.12.188.

Oliver, J.V.; Abián, M.A. 2013. Advanced wireless sensors for termite detection in wood constructions. Wood Sci Technol 47: 269-280. https://doi.org/10.1007/s00226-012-0485-8.

Osuna-Sequera, C. 2017. Particularidades de la aplicación de las técnicas no destructivas en la estimación de propiedades mecánicas en piezas de madera de gran longitud procedentes de estructuras existentes (Considerations on non-destructive testing applied to estimate mechanical properties in large length timber pieces from existing structures). Final Project Degree. UPM, Madrid, Spain. http://oa.upm.es/51760/.

Osuna-Sequera, C.; Íñiguez-González, G.; Esteban, M.; Llana, D.F.; Arriaga, F. 2017. Particularidades de la aplicación de las técnicas no destructivas en piezas de madera de gran longitud procedentes de estructuras existentes (Considerations on the application of nondestructive testing on large length timber pieces from existing structures). In Proceedings of LIGNOMAD 17 the $1^{\circ}$ Congreso sobre Construcción con Madera y otros Materiales Lignocelulósicos. Barcelona, Spain. pp. 53-57.

Osuna-Sequera, C.; Llana, D.F.; Íñiguez-González, G.; Arriaga, F. 2019a. The influence of cross-section variation on bending stiffness assessment in existing timber structures. Eng Struct. 204(2020): 110082. https://doi.org/10.1016/j.engstruct.2019.110082.

Osuna-Sequera, C.; Llana, D.F.; Esteban, M.; Arriaga, F. 2019b. Improving density estimation in large cross-section timber from existing structures by increasing the number of non-destructive measurements. Constr Build Mater 211: 199-206. https://doi.org/10.1016/j.conbuildmat.2019.03.144.

Palaia, L.; Galvañ, V.; Cervera, F.; Monzo, V. 1993. Using ultrasonic waves for the detection of timber decay in old buildings. In Proceedings of the $9^{\text {th }}$ International Symposium on Nondestructive Testing of Wood. Madison, WI, USA. pp. 71-77.

Palaia, L.; Galvañ, V.; Cervera, F.; Molina, R. 2000. NDT determination of the strength loss of deteriorated wood, by using ultrasonic techniques and a specific wood tester. In Proceedings of the $12^{\text {th }}$ International Symposium on Nondestructive Testing of Wood. Sopron, Hungary. pp. 327-336.

Palaia, L.; Monfort, J.; Sánchez, R.; Gil, L.; Álvarez, Á.; López, V.; Tormo, S.; Pérez, C.; Navarro, P. 2008. Assessment of timber structures in service by using combined methods of non-destructive testing together with traditional ones. In Proceedings of the $9^{\text {th }}$ International Conference on NDT of Art. Jerusalem, Israel. $10 \mathrm{p}$.

Pedras, F.; Riesco G.; Remacha, A. 1997. Determinación de las características elásticas de la madera de castaño (Castanea sativa Mill.) mediante ensayos no destructivos (Determination of elastic properties of sweet chestnut (Castanea sativa Mill.) by NDT). In Proceedings of the $2^{\circ}$ Congreso Forestal Español. Pamplona, Spain. pp. 295-300.

Pérez-García, A. 2012. Estimación de parámetros de calidad sobre tableros derivados de la madera a partir de ensayos no destructivos (Wood-board quality parameter estimation by non-destructive testing). Final Project Degree. UPM, Madrid, Spain. http://oa.upm.es/11295/.

Riesco, G. 2001. Estudio de las propiedades físico-mecánicas de la madera de roble (Quercus robur L.) de Galicia en relación con las variables del medio (Study of physico-mechanical properties of European oak (Quercus robur L.) timber from Galicia in relation with environmental variables). Ph.D. Thesis, UPM, Madrid, Spain.

Rodríguez-Abad, I.; Martínez-Sala, R.; Capuz, R.; Díez, M.R.; García-García, F. 2011. Estudio de la variación del contenido de humedad en el Pinus pinaster Ait. por medio de la técnica no destructiva del geor- 
radar (Assessment of the variation of the moisture content in Pinus pinaster Ait. using the non destructive GPR technique). Mater Construcc 61(301): 143-156. https://doi.org/10.3989/mc.2010.49608.

Rodríguez-Liñán, C.; Rubio, P. 1995. Evaluación del estado de la madera, en obras de rehabilitación, mediante técnicas de ultrasonidos y obtención de parámetros resistentes (Evaluation of wood conservation works by ultrasound techniques and obtention of resistance parameters). Inf Constr 47(440): 5-22. https://doi. org/10.3989/ic.1995.v47.i440.1044.

Rodríguez-Liñán, C.; Rubio, P. 2000. Evaluación del estado de la madera, en obras de rehabilitación, mediante técnicas de ultrasonidos (Timber assessment by ultrasound techniques in refurbishment works). Universidad de Sevilla, Secretariado de Publicaciones, Instituto Universitario de Ciencias de la Construcción: Sevilla, Spain. https://expobus.us.es/omeka/items/show/883.

Rojas, J.A.M.; Alpuente, J.; Postigo, D.; Rojas, I.M.; Vignote, S. 2011. Wood species identification using stress-wave analysis in the audible range. Appl Acoust 72: 934-942. https://doi.org/10.1016/j.apacoust.2011.05.016.

Ruano, A.; Zitek, A.; Hinterstoisser, B.; Hermoso, E. 2019. NIR hyperspectral imaging (NIR-HI) and $\mu \mathrm{XRD}$ for determination of the transition between juvenile and mature wood of Pinus sylvestris L. Holzforschung 73(7): 621-627. https://doi.org/10.1515/hf-2018-0186.

Rubio, P. 1997. Evaluación del estado de la madera mediante técnicas de ultrasonidos (Assessment of timber by ultrasound techniques). Ph.D. Thesis. US, Sevilla, Spain. http://hdl.handle.net/11441/23937.

Salamanca, M. 2017. Estimación mediante penetrómetros de la densidad en madera aserrada de las especies de coníferas y frondosas más habituales en construcción en España (Study on the estimation, by penetrometers, of the density of sawn wood of the most common coniferous and leafy species used in construction in Spain). Final Project Degree. UPM, Madrid, Spain.

Sánchez-Beitia, S.; Crespo-de-Antonio, M.; Acuña, L. 2015. Applicability of the Hole-Drilling procedure for stresses quantification in timber bending elements in service. Constr Build Mater 93: 798-805. https:// doi.org/10.1016/j.conbuildmat.2015.05.091.

Santaclara, O.; Fernández, A.; Merlo, E.; Guaita, M. 2009. Técnicas no destructivas y ensayo mecánico en madera aserrada de gran escuadría de Pseudotsuga menziesii (Nondestructive testing and mechanical testing of large cross-section Pseudotsuga menziesii sawn timber). In Proceedings of the V Congreso Nacional y II Congreso Ibérico AgroIngeniería. Lugo, Spain. 9 p.

Santaclara, O.; Merlo, E. 2011. Acoustic segregation of Pinus pinaster logs for structural lumber production according to strength classes. In Proceedings of the $17^{\text {th }}$ International Nondestructive Testing and Evaluation of Wood Symposium. Sopron, Hungary. p. 755.

Sevilla, S.; Bobadilla, I.; Martínez, R.D.; Esteban, M.; Llana, D.F. 2013. Non-destructive methods to estimate the physical aging of Superpan. In Proceedings of the $18^{\text {th }}$ International Nondestructive Testing and Evaluation of Wood Symposium. Madison, WI, USA. p. 637.

Soto-Martínez, I. 2010. Estudio de la relación entre perfiles de densidad y gráficas resistográficas en madera estructural y aplicaciones industriales (Comparison between density and resistograph results in structural timber and industrial applications). Final Project Degree. UPV, Gandía, Spain. https://riunet.upv.es/handle/10251/9983.

Touza, M.C. 2009. Case study: Assessment of the mechanical properties of an old pitch pine timber structure. In Proceedings of the International Conference on Wooden Cultural Heritage. Hamburg, Germany. 8 p.

Touza, M.C.; Soilán, A.; Lorenzo, D. 2013. Evaluation of the load-carrying capacity in bending of large cross section "Pitch pine" beams in standing structures. Adv Mater Res-Switz 778: 410-417. https://doi. org/10.4028/www.scientific.net/AMR.778.410.

Troya, M.T.; Navarrete, A. 1994. Study of the degradation of retified wood through ultrasonic and gravimetric techniques. In Proceedings of the $25^{\text {th }}$ Annual meeting. Bali, Indonesia. 6 p. 
UNE. 1972. Clasificación de la madera aserrada de construcción. UNE 56525. 1972. (Visual grading of construction sawn timber). IRANOR/CTN 56 Madera y Corcho: Madrid, Spain.

UNE. 2011. Clasificación visual de la madera aserrada para uso estructural. Madera de coníferas. UNE 56544. 2011. (Visual grading for structural sawn timber. Coniferous timber). AEN/CTN 56 Madera y Corcho: Madrid, Spain.

UNE. 2013. Clasificación visual de la madera aserrada para uso estructural. Madera de frondosas. UNE 56546. 2013. (Visual grading for structural sawn timber. Hardwood timber). AEN/CTN 56 Madera y Corcho: Madrid, Spain.

UNE. 2014. Estructuras de madera existentes. Uso del penetrómetro para el diagnóstico de los elementos de madera de edificios existentes. UNE 41809. 2014. (Existing wood structures. Use of penetrometer in wood elements diagnosis in existing buildings). AEN/CTN 41 Construcción: Madrid, Spain.

UNE. 2018. Clasificación visual de los postes de madera para líneas aéreas. UNE 56547. 2018. (Visual classification for wood overhead poles). CTN 56 Madera y Corcho: Madrid, Spain.

Vázquez, C.; Gonçalves, R.; Bertoldo, C.; Baño, V.; Vega, A.; Crespo, J.; Guaita, M. 2015. Determination of the mechanical properties of Castanea sativa Mill. using ultrasonic wave propagation and comparison with static compression and bending methods. Wood Sci Technol 49: 607-622. https://doi.org/10.1007/ s00226-015-0719-7.

Vega, A.; Dieste, A.; Guaita, M.; Majada, J.; Baño, V. 2012. Modelling of the mechanical properties of Castanea sativa Mill. structural timber by a combination of nondestructive variables and visual grading parameters. Eur J Wood Prod 70(6): 839-844. https://doi.org/10.1007/s00107-012-0626-7.

Vega, A. 2013. Caracterización mecánica de la madera estructural de Castanea sativa Mill. clasificación visual y evaluación mediante métodos no destructivos (Mechanical characterization of Castanea sativa Mill. structural sawn timber: visual grading and nondestructive testing evaluation). Ph.D. Thesis. USC, Lugo, Spain. https://minerva.usc.es/xmlui/handle/10347/9345.

Vega, A.; Arriaga, F.; Guaita, M.; Baño, V. 2013. Proposal for visual grading criteria of structural timber of sweet chestnut from Spain. Eur J Wood Prod 71: 529-532. https://doi.org/10.1007/s00107-013-0705-4.

Vega, A.; González, L.; Fernández, I.; González, P. 2019a. Grading and mechanical characterization of small-diameter round chestnut (Castanea sativa Mill.) timber from thinning operations. Wood Mater Sci Eng 14(2): 81-87. https://doi.org/10.1080/17480272.2017.1387174.

Vega, A.; González, L.; Rodríguez, S.; Hevia, A. 2019b. Prediction of the sawing yield of Pinus pinaster and Pinus radiata structural timber from thinning operations through Nondestructive Techniques. In Proceedings of the $21^{\text {st }}$ International Nondestructive Testing and Evaluation of Wood Symposium. Freiburg im Breisgau, BW, Germany. pp. 577-583.

Vilches, M.; Correal, E. 2009. Aplicación del resistógrafo para la determinación de las propiedades de la madera (Using a resistograph to determine wood properties). In Proceedings of the $5^{\circ}$ Congreso Forestal Español. Avila, Spain. 8 p.

Vilches, M.; Labèrnia, C.; Rodríguez, V. 2015. Assessment through NDT of the state of timber structures of the historic buildings of Catalonia. COST Action FP 1101 Assessment, reinforcement and monitoring of timber structures. pp 209-216.

Villacorta-Calvo, J.J.; Izquierdo-Fuente, A.; Val-Puente, L.; Suárez, L.; Martínez, R.D.; Morillas, L.; Basterra, L.A. 2019. AmemoMe: Diseño e implementación de un sistema de medida basado en acelerómetros MEMS para el análisis de los modos de vibración de una estructura de madera (AmemoMe: Accelerometers MEMS measurement system design and implementation to analyze vibration modes on a timber structure). In Proceedings of LIGNOMAD 19 the $2^{\circ}$ Congreso sobre Construcción con Madera y otros Materiales Lignocelulósicos. Santiago de Compostela, Spain. pp. 72-76.

Villanueva, J.L. 2009. Caracterización mecánica de rollizos de sabina (Juniperus thurifera L.) de Castilla y León. Prueba de clasificación visual y evaluación mediante resonancia (Mechanical characterization 
of Spanish juniper (Juniperus thurifera L.) round timber from Castilla y León. Visual grading and resonance evaluation). Final Project Degree. UdL, Lérida, Spain. https://repositori.udl.cat/handle/10459.1/45926.

Villasante, A.; Íñiguez-González, G.; Puigdomenech, L. 2019. Comparison of various multivariate models to estimate structural properties by means of non-destructive techniques (NDTs) in Pinus sylvestris L. timber. Holzforschung. 73(4): 331-338. https://doi.org/10.1515/hf-2018-0103. 